Journal for ImmunoTherapy of Cancer \title{
antibody to redirect $T$ cells for cancer immunotherapy \\ Novel potent anti-STEAP1 bispecific
}

To cite: Lin T-Y, Park JA, Long A et al. Novel potent anti-STEAP1 bispecific antibody to redirect $T$ cells for cancer immunotherapy. Journal for ImmunoTherapy of Cancer 2021;9:e003114. doi:10.1136/jitc-2021-003114

- Additional supplemental material is published online only. To view, please visit the journal online (http://dx.doi.org/10. 1136/jitc-2021-003114).

T-YL and JAP contributed equally.

T-YL and JAP are joint first authors.

Accepted 17 August 2021

\section{Check for updates}

(C) Author(s) (or their employer(s)) 2021. Re-use permitted under CC BY-NC. No commercial re-use. See rights and permissions. Published by BMJ.

Pediatrics, Memorial Sloan Kettering Cancer Center, New York, New York, USA

Correspondence to Dr Nai-Kong V Cheung; cheungn@mskcc.org

Tsung-Yi Lin, Jeong A Park (D) , Alan Long, Hong-Fen Guo, Nai-Kong V Cheung

\section{ABSTRACT}

Background The prognosis for metastatic Ewing sarcoma family of tumors (EFT) is still poor despite high-dose chemotherapy and radiation treatment. Immunotherapies hold promise, but cancer antigen-targeting immunotherapies have largely failed to induce effective T cell receptor-mediated antitumor response. However, $\mathrm{T}$ cell-engaging bispecific antibodies (T-BsAbs) have yet to be adequately explored.

Methods Rehumanized STEAP1-IgG was used to build T-BsAb (named BC261) using the 2+2 IgG-[L]-scFv platform carrying the anti-CD3 huOKT3 scFv as the second specificity. Its binding epitope mapping, species cross-reactivity, tumor cell line staining, and in vitro cytotoxicity were investigated thoroughly. Its potency in driving tumor-infiltrating lymphocytes (TILs) was quantified using bioluminescence, correlated with in vivo antitumor response against cell line-derived or patientderived xenografts (CDXs or PDXs) and compared with anti-STEAP1 T-BsAbs built on representative antibody platforms.

Results BC261 binding epitope was mapped to its second extracellular domain of STEAP1 shared among canine and primate orthologs. BC261 induced potent cytotoxicity against panels of EFT, prostate cancer, and canine osteosarcoma cell lines despite their low antigen density. BC261 drove significantly more TILs into tumors (30-fold) and exerted superior antitumor effects compared with the other standard BsAb platforms. The antitumor efficacy of BC261 was consistent against EFT and prostate cancer CDXs and PDXs.

Conclusions BC261 was highly efficient in driving T cell infiltration and tumor ablation. Either as stand-alone therapeutics or for ex vivo armed T cells, this novel antiSTEAP1 T-BsAb BC261 has therapeutic potential.

\section{INTRODUCTION}

Ewing sarcoma family of tumors (EFT), which includes Ewing sarcoma of bone, extraosseous Ewing tumor, and peripheral primitive neuroectodermal tumor, is the second most common malignant bone tumor in children and young adults. ${ }^{12}$ EFT is characterized by the oncogenic EWS-ETS translocation including EWS-FLI1, t(11;22) (q24;q12) and EWS-ERG, t(21;22) (q11;q12) and early metastasis. $^{3-5}$ For localized EFT, cytotoxic chemotherapy has improved the survival from less than $20 \%$ to $70 \%-80 \%$. However, over the last three decades, there has been a modest improvement in survival, and the prognosis for metastatic or relapsed disease has remained poor, with survival rates of less than 20\%-30\%. ${ }^{67}$ Given the high toxicities associated with intensive chemotherapy and radiotherapy, there is an urgent unmet need for novel therapeutic approaches, and $\mathrm{T}$ cell-based immunotherapies are promising alternatives. 89 However, despite the tumor specificity of EWS-FLI1 fusion sequences, the patients failed to induce strong cytotoxic $\mathrm{T}$ cell response because of low MHC expression and defective antigen processing in EFT. ${ }^{10} 11$ Cancer/testis antigens including membraneassociated phospholipase A1 beta (LIPI), ${ }^{12}$ $\mathrm{X}$ antigen family member 1 (XAGE1), ${ }^{13}$ and New York esophageal squamous cell carcinoma 1 (NY-ESO-1) ${ }^{14}$ have also been considered as promising targets for immunotherapy with the advantage of low expression in adult somatic tissues, but the clinical trials have yielded limited efficacy. ${ }^{15} 16$

The six-transmembrane epithelial antigen of prostate (STEAP) family of proteins which include four members, named STEAP1 to STEAP4, are overexpressed in several human cancers. ${ }^{17}$ These STEAP proteins, particularly STEAP1 and STEAP2 that control cell proliferation and apoptosis, have potential as therapeutic targets. ${ }^{18}$ However, except for STEAP1, the relationship between overexpression of these other STEAP proteins and tumor aggressiveness has remained largely elusive. $^{20-22}$ STEAP1 is upregulated in a variety of human cancers, including prostate, bladder, ovarian, rhabdomyosarcoma, and EFT. ${ }^{1921}$ The expression of STEAP1 in normal tissues (except secretory tissues of the bladder and prostate) is highly restricted, ${ }^{23}{ }^{24}$ suitable for antibody-based immunotherapies. ${ }^{25}$ 26 Several therapeutic strategies targeting STEAP1 have been developed including monoclonal antibodies, antibody drug 
conjugates, DNA vaccines, and small non-coding RNAs for the treatment of prostate cancers. ${ }^{27}$ Radiolabeled antiSTEAP1 monoclonal antibody, ${ }^{89} \mathrm{Zr}$-DFO-MSTP2109A (NCT01774071), confirmed its highly restricted tumor tissue distribution, ${ }^{28}{ }^{29}$ and antibody-drug (MMAE) conjugate targeting STEAP1 (DSTP3086S) has shown acceptable safety profiles with a modest clinical benefit in patients with metastatic castration-resistant prostate cancer. ${ }^{26}$ With the advent of T cell-based immunotherapy, $\mathrm{T}$ cell engaging bispecific antibodies (T-BsAb) or chimeric antigen receptor (CAR) T cells targeting STEAP1 also are early in human trials. ${ }^{30-34}$ But most of these therapeutic trials are still in the beginning stage, and the most optimal platforms have yet to emerge.

We previously demonstrated that the interdomain distance and cis-configuration are critical for in vivo antitumor response of T-BsAb. ${ }^{35}$ We also showed that a $2+2$ format (2 tumor binding domains+2 CD3 binding domains) was substantially more potent than a $2+1$ format (2 tumor binding domains+1 CD3 binding domain). ${ }^{35} 36$ Here, we describe the biochemical and cytotoxic properties of the STEAP1 BsAbs built on the IgG-[L]-scFv format, their epitope mapping, species cross reactivity, and their in vivo antitumor activities against a panel of EFT and prostate cancer xenografts.

\section{METHODS}

\section{Humanization of murine anti-STEAP1 antibody X120}

The anti-STEAP1 monoclonal antibody X120 $0^{37}$ was rehumanized by grafting complementarity-determining regions (CDRs) onto human IgG1 frameworks based on their homology with human frameworks IGHV4-30-4*01IGHJ $6 * 01$ for variable heavy chain $(\mathrm{VH})$, IGKV4-1*01IGKJ $4 * 01$ for variable light chain (VL), respectively. Six humanized $\mathrm{VH}$ (VH1 to VH6) and four humanized VL (VL1 to VL4) sequences were paired to create 24 humanized versions, with identical CDR sequences to the mouse antibody X120 ${ }^{37}$ using $\mathrm{CHO}$ cells. Instead of surface plasmon resonance analysis, mean fluorescence intensity (MFI) of antibody binding to EFT cell line TC-32 was used to rank binding affinity of 24 antibodies due to conformational epitope of the STEAP1 (online supplemental figure 1A), and eight IgG clones pairing VH1 or VH2 with VL1 to VL4 and VH5 +VL1 were chosen for binding avidity test. After eight cycles of repeated washing using phosphate buffered saline (PBS/EDTA dissociation buffer, four clones (VH1 +VL1, VH2 +VL1 and VH2 +VL2, and VH5 +VL1) retained $>50 \%$ of their baseline MFIs, higher than that of the chimeric antibody (online supplemental figure 1B). Binding affinities of these four clones were compared with that of the chimeric antibody using a cell-based fluorescent method ${ }^{38}$ (online supplemental table 1). Antibody purity was analyzed by size-exclusion high-performance liquid chromatography (SEC-HPLC), and antibody stability was monitored by SEC-HPLC after freeze-thaw cycles or after heat stress at $40^{\circ} \mathrm{C}$ for 4 weeks. These four clones with $>85 \%$ sequence humanness showed high binding affinities to targets and high purity and stability after multiple freeze-thaw cycles or heat stress at $40^{\circ} \mathrm{C}$ over 4 weeks when assayed by HPLC (online supplemental table 2) and were chosen for developing T-BsAb.

\section{Bispecific antibodies}

Four anti-STEAP1 T-BsAbs (named BC259, BC260, BC261, BC262) with different VH and VL sequences were developed using the IgG-[L]-scFv platform ${ }^{31}{ }^{40}$ by attaching the anti-CD3 humanized OKT3 (huOKT3) single-chain variable fragment $(\mathrm{scFv})$ to the carboxyl end of the rehumanized X120 IgG1 light chain. The amino acid sequences for the VH and VL regions for these bispecific antibodies are summarized in online supplemental table 3. Each BsAb was produced using the Expi293 expression system (Thermo Fisher Scientific RRID:CVCL_D615) or HEK 293 T cell (RRID:CVCL_KS61) and maintained in $\mathrm{pH} 8.250 \mathrm{mM}$ of citrate buffer with $150 \mathrm{mM}$ of NaCl. The lead clone BC261 was expressed in CHO cells (RRID:CVCL_0213). Antibodies were purified with protein A affinity column chromatography, and the stability was monitored by SEC-HPLC weekly for 4 weeks at $40^{\circ} \mathrm{C}$.

\section{Tumor cell lines}

EFT cell lines, TC-32 (RRID:CVCL_7151), TC71 (RRID:CVCL_2213), SK-ES-1 (RRID:CVCL_0627), SK-NMC(RRID:CVCL_0530), and A4573 (RRID:CVCL_6245) were purchased from ATCC; SKEAW, SKELP, SKERT were provided by Memorial Sloan Kettering Cancer Center (MSKCC) investigators. Prostate cancer cell lines, CWR22 (RRID:CVCL_3967), LNCaP-AR (CLS Cat\# 300265/p761_LNCaP, RRID:CVCL_0395), and VCaP (RRID:CVCL_2235) were purchased from ATCC. Canine osteosarcoma cell lines, D-17 (RRID:CVCL_1916), DAN (RRID:CVCL_1917), DSN (RRID:CVCL_1919), and DSDh (RRID:CVCL_1918) were purchased from ATCC. All cancer cells were authenticated by short tandem repeats profiling using PowerPlex 2.1 System (Promega, Cat\# DC8942), and periodically tested for mycoplasma infection using a commercial kit (Lonza, Cat\# LT07-318).

\section{Flow cytometry analysis of STEAP1 antibody binding}

For STEAP1 density measurement on tumor cells, antiSTEAP1 human IgG1 was chemically conjugated to AlexFluor 647 (Thermo Fisher) according to manufacturer's instructions and used to stain STEAP-1 BsAbs bound to human cancer cells. Confirmatory binding to targets was analyzed using anti-human IgG Fc antibody. For quantitation of target antigens, MFI was translated into antigen density using Quantum MESH microspheres and fluorochrome to protein ratio (Bangs Laboratories, cat\# 647). One microgram of STEAP1 IgG monoclonal antibodies or STEAP1 BsAbs were incubated with $1 \times 10^{6}$ of cancer cells at $4^{\circ} \mathrm{C}$ for $45 \mathrm{~min}$, washed, reacted with R-phycoerythrin conjugated goat anti-human IgG secondary antibody (SouthernBiotech Cat\#2040-09, RRID:AB_2795648), 
and analyzed by flow cytometry (BD FACSCalibur instrument and Attune NxT Flow Cytometer). Palivizumab or anti-CD33 BsAb was used as a control antibody. Data were analyzed with FlowJo V.10 software (FlowJo, RRID:SCR_008520).

\section{T cell expansion ex vivo}

Peripheral blood mononuclear cells (PBMCs) were separated from buffy coats (New York Blood Center) by Ficoll. Naive $\mathrm{T}$ cells were purified from human PBMC using Pan T cell isolation kit (Miltenyi Biotec, Cat\#130096535) and expanded by CD3/CD28 Dynabeads (Gibco, Cat\#11 132D) for 7-14 days in the presence of $30 \mathrm{IU} / \mathrm{mL}$ of IL-2 according to manufacturer's instructions. Unless stated otherwise, these activated $\mathrm{T}$ cells were used for all $\mathrm{T}$ cell experiments.

\section{Cytotoxicity assay (chromium ${ }^{51}$ release assay)}

In vitro antibody-dependent $T$ cell-mediated cytotoxicity assay was carried out as previously described. ${ }^{35} 41$ Target cells were cultured in RPMI1640 (Cellgro) supplemented with $10 \%$ fetal bovine serum (Life Technologies) at $37^{\circ} \mathrm{C}$ in a $5 \% \mathrm{CO}_{2}$ humidified incubator. Target cells were labeled with sodium ${ }^{51} \mathrm{Cr}$ chromate $(51 \mathrm{CrNa} 2 \mathrm{CrO} 4$; Amersham, Arlington Heights, Illinois, USA) at $100 \mu \mathrm{Ci} / 10^{6}$ cells at $37^{\circ} \mathrm{C}$ for 1 hour. Percentage of specific lysis was calculated using the formula $100 \% \mathrm{x} \times$ (experimental cpmbackground $\mathrm{cpm}) /($ total cpm-background cpm), where cpm represented counts per minute of ${ }^{51} \mathrm{Cr}$ released. Total release was assessed by lysis with 10\% SDS (Sigma, St Louis, Missouri, USA), and background release was measured in the absence of effector cells. Glycoprotein A33 (GPA33) or anti-CD33 BsAb was used as a control BsAb.

\section{STEAP1 transduction through lentivirus}

Lentiviruses were produced by transfection of $2 \times 10^{7} 293 \mathrm{~T}$ cells using $20 \mu \mathrm{g}$ of lentiviral vector pLVX-IRES-ZsGreen 1 (Takara,Cat\#632187), $10 \mu \mathrm{g}$ of pVSV-G (RRID:Addgene_138479), $20 \mu \mathrm{g} \quad$ pCMV-dR8.91 (Lifescience Market), and $100 \mu \mathrm{L}$ PolyJet In Vitro DNA Transfection Reagent (SignaGen, Cat\#SL100688, Rockville, MD) as described previously. ${ }^{42} 43$ After STEAP1 variants transduction, cells were sorted by ZsGreen1 GFP fluorescence.

\section{T cell transduction with luciferase and tdTomato}

$\mathrm{T}$ cells isolated from peripheral blood were stimulated with Dynabeads Human T-Activator CD3/CD28 for 24 hours. T cells were transduced with retroviral constructs containing tdTomato and click beetle red luciferase in RetroNectin-coated 6-well plates in the presence of IL-2 $(100 \mathrm{IU} / \mathrm{mL})$ and protamine sulfate $(4 \mu \mathrm{g} / \mathrm{mL})$ as previously described. ${ }^{44}$ Transduced T cells were cultured for 8 days before being used in animal experiments.

\section{Bioluminescence imaging}

To monitor $\mathrm{T}$ cell trafficking into tumors, BsAb armed luciferase transduced $\mathrm{T}$ cells were injected intravenously. Mice were anesthetized and imaged after intravenous injection of $3 \mathrm{mg}$ of D-luciferin (Gold Biotechnology, Cat\# LUCK-100) on different days post $\mathrm{T}$ cell injection. Images were acquired using IVIS Spectrum CT In Vivo Imaging System (Caliper Life Sciences). Bioluminescence images were overlaid with photographs, and regions of interest were drawn based on the location and contour of tumor using Living image 2.60 (Xenogen). Quantification of bioluminescence emission (BLI, photon flux/sec) was performed, monitored by time, and compared with the BLIs of tumors without treatment.

\section{Cytokine release assays}

Human Th1 cell-released cytokines were analyzed after EAT injection using LEGENDplex Human Th1 Panel (Biolegend, Cat\# 741035). Five human $\mathrm{T}$ cell cytokines including IL-2, IL-6, IL-10, IFN- $\gamma$, and TNF- $\alpha$ were analyzed using mouse serum after EAT injection.

\section{In vivo studies}

All animal experiments were performed in compliance with MSKCC Institutional Animal Care and Use Committee (IACUC) guidelines. BALB $/ \mathrm{cRag}^{-/-} \mathrm{IL}-$ $2 \mathrm{R} \gamma \mathrm{c}^{-/-}$(BRG) mice were purchased from Taconic Biosciences (Hudson, New York, USA) and provided with sulfatrim or amoxicillin food. Two EFT patient-derived tumor xenografts (PDXs), ES03a and ES15a, were established from surgical specimens by the antitumor core facility in MSKCC with MSKCC IRB approval, and prostate cancer PDXs, TM00298 and J000077451, were purchased from The Jackson Laboratory. Tumor cells were suspended in Matrigel Basement Membrane Matrix (Corning, Cat\#354234) and implanted in the flank of 6-week-old to 10-week-old BRG mice; only male mice were used for prostate cell line-derived xenografts (CDXs) and PDXs. Treatment was initiated after tumors were established, average tumor volume of $100 \mathrm{~mm}^{3}$ when measured using TM900 scanner (Piera, Brussels, BE) after 8 days to 2 months post subc tumor implantation. Before treatment, mice with small tumors $\left(<50 \mathrm{~mm}^{3}\right)$ or infection signs were excluded from randomization to experimental groups. For treatment, BsAb and human $\mathrm{T}$ cells were intravenously (iv) injected separately or together (ex vivo armed T cells) with supplementary subcutaneous IL-2 (1000 IU). Ex vivo armed T cells (EATs) were generated by incubating $\mathrm{T}$ cells with $\mathrm{T}-\mathrm{BsAb}$ for $20 \mathrm{~min}$ at room temperature. After incubation, the $\mathrm{T}$ cells were washed with PBS twice. The T cell number administered per dose was $2 \times 10^{7}$ cells based on previous reports. ${ }^{45}$ For in vivo experiment, anti-GPA33 $\mathrm{BsAb}$ was used as a control BsAb. Tumor growth curves and overall survival curves were plotted, and the overall survival was defined as the time from start of treatment to when tumor volume reached $2000 \mathrm{~mm}^{3}$. To define wellbeing of mice, $\mathrm{CBC}$ analyses, changes in body weight, general activity, physical appearance, and graft-versushost disease (GVHD) scoring were regularly monitored. When tumor growth reached $2000 \mathrm{~mm}^{3}$ or greater, mice were euthanized. All animal experiments were repeated 
twice more with different donor's $\mathrm{T}$ cells to ensure that our results were reliable.

\section{Statistical analyses}

Tumor growth rates and bioluminescence of $\mathrm{T}$ cells were analyzed using area under the curve (AUC). The AUC and overall survival were calculated using GraphPad Prism V.8.0. Difference between each treatment group was tested for statistical significance by two-tailed Student's t-test for two sets of data while one-way analysis of variance with Tukey's post hoc test was used among three or more groups. All statistical analyses were performed using GraphPad Prism V.8.0 for Windows (GraphPad Software, La Jolla, California, USA, www. graphpad. com). $\mathrm{P}$ value $<0.05$ was considered statistically significant. Asterisks indicate that the experimental $p$ value is statistically significantly different from the associated controls at $* \mathrm{p}<0.05 ; * * \mathrm{p}<0.01 ; * * * \mathrm{p}<0.001, * * * * \mathrm{p}<0.0001$.

\section{RESULTS}

\section{STEAP1 BsAb design and expression in Expi293 cells}

Four STEAP1 BsAbs, that is, BC259 (VH1 +VL1), BC260 (VH2 +VL1), BC261 (VH2 +VL2), BC262 (VH5 +VL1), were built using the $2+2 \mathrm{IgG}-[\mathrm{L}]-\mathrm{scF}$ v BsAb format. The representative platform for these 4 STEAP1 BsAbs is described in figure 1A. CH1 through CH3 and CL were based on constant domains of the heavy chain and of kappa light chain of the humanized anti-STEAP1 IgG antibody. The C-terminus of the CL is fused to the scFv of an anti-CD3 humanized OKT3 (huOKT3). The biochemical purity of BC261, representative of four clones, was analyzed by SEC-HPLC (online supplemental figure 2A), where the elution fractions were confirmed by sodium dodecyl sulfate-polyacrylamide gel electrophoresis (SDSPAGE). All four STEAP1 BsAbs remained stable after multiple freeze-thaw cycles and heat stress at $40^{\circ} \mathrm{C}$ over 4 weeks (online supplemental table 4 ), and among them, BC261 maintained high purity despite prolonged heat stress.

\section{STEAP1 BsAb binding to EFT and prostate cancer cell lines}

STEAP1 BsAb binding to EFT cell lines was analyzed by flow cytometry. All four BsAbs showed reactivity against a panel of EFT cell lines (TC-32, TC71, SK-ES-1, and A4573) and prostate cancer cell line (LNCaP-AR) (online supplemental figure 2B). While BC261 MFI was consistently the highest, BC262 MFI was the lowest among the four BsAbs. Immunostaining of EFT by BC261 was expanded to more EFT cell lines (SKEAW, SKERT, SKELP, SKNMC, and TC71Luc), and STEAP1 antigen expression was quantified as molecules per cell, confirming the relatively low density of the STEAP1 compared with other target antigens (figure 1B). After screening a large panel of human or non-human cancer cell lines (online supplemental table 5), STEAP1 BsAb was found to bind prostate cancer cell lines and canine osteosarcoma cell lines as well, and the expression density was quantified (figure 1C).

\section{STEAP1 BsAb-dependent T cell-mediated cytotoxicity}

In vitro cytotoxicity of STEAP1 BsAb was tested against a panel of EFT, using HER2-BsAb ${ }^{32}$ as a positive control and anti-GPA33 BsAb $^{46}$ as a negative control. BC259 and BC261 showed robust cytotoxicity against TC-32, TC71, and SK-ES-1 cell lines (figure 1D), while BC262 was much less potent. The in vitro potency of STEAP1 BsAb as measured by EC50 of BC261 against A4573, SKEAW, SKELP, SKERT, and SKNMC ranged between 1.1 and 25.8 pM (online supplemental table 6); against prostate cancer cell lines LNCaP-AR, CWR22, and VCaP between 21.1 and $214.8 \mathrm{pM}$ (figure 1E); and against canine osteosarcoma D-17, DSN, DAN, and DSDh between 7.2 and $29.5 \mathrm{pM}$ (online supplemental figure 3A). These EC50s correlated with the levels of STEAP1 expression measured by MFI (online supplemental table 6).

\section{In vivo antitumor effect of STEAP1 BsAbs}

In vivo antitumor efficacy of these 4 STEAP1 BsAbs was evaluated against TC-32 CDXs. Once tumors were established, STEAP1 BsAbs were administered with human T cells twice per week for 2-3 weeks (figure 2A). In contrast to control groups which showed unabated tumor growth, all four STEAP1 BsAbs exerted significant antitumor effects $(p<0.0001)$. The mice developed mild to moderate GVHD between 4 weeks and 8 weeks after treatment start, but recovered spontaneously, showing no obvious toxicity through 1 year after the start of treatment. There were no recurrences except for two mice in the BC262 (VH5 +VL1) treated group. Since BC261 (VL2 +VH2) had the greatest humanness $(>85 \%)$, reacted with EFT cell lines with higher MFIs, possessed highest stability at $40^{\circ} \mathrm{C}$, and showed the most consistent antitumor effect in vitro and in vivo, it was chosen for further investigations. BC261 achieved exceptional antitumor efficacy against large solid tumors (figure 2B). Even for large established tumors $\left(\sim 2000 \mathrm{~mm}^{3}\right)$, BC261 achieved complete tumor regression, an effect not typically seen with T-BsAbs targeting other tumor antigens (eg, GD2, ${ }^{39}$ HER2, ${ }^{32}$ or GPA33 ${ }^{46}$ ) even when built on the same IgG-[L]-scFv platform.

\section{Analysis of STEAP1 epitope for BC261}

The parental mouse antibody and the humanized antiSTEAP1 IgG did not react with mouse cell lines. ${ }^{37}$ Given the sequence homology between human and canine STEAP1 of $90.56 \%$ and between human and mouse STEAP1 of $82.01 \%$ (278/339 aa), analyses of the extracellular domain (ECD) sequences of these STEAP1 orthologs suggested an epitope on the second ECD (figure 3A). Four STEAP1 variants, human STEAP1 (STP1h), mouse STEAP1 (STP1m), mouse STEAP1 with human second ECD (STP1mH2), and mouse STEAP1 with human third ECD (STP1mH3), were constructed and transfected into STEAP1(-) HEK293T cells using lentivirus. After sorting by the expression of ZsGreen1 GFP reporter protein (figure 3B), the STEAP1 transfectants were stained with BC261. Only the STP1mH2 variant showed comparable 
(A)

\section{$2+2 \lg G-[L]-s c F v$}

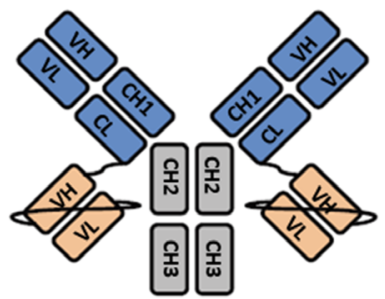

(B)

(C)

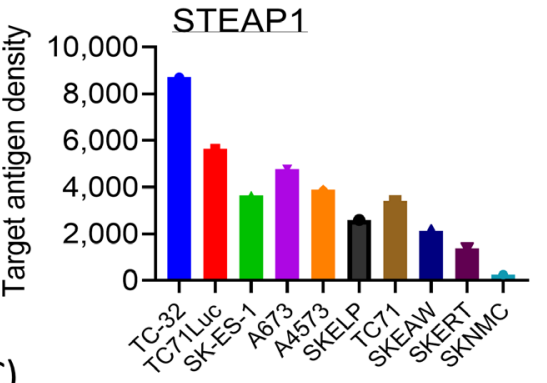

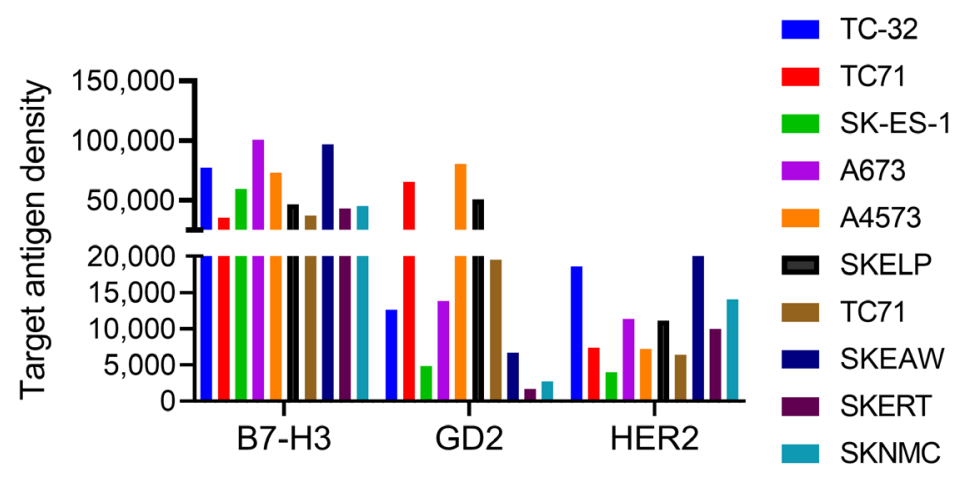
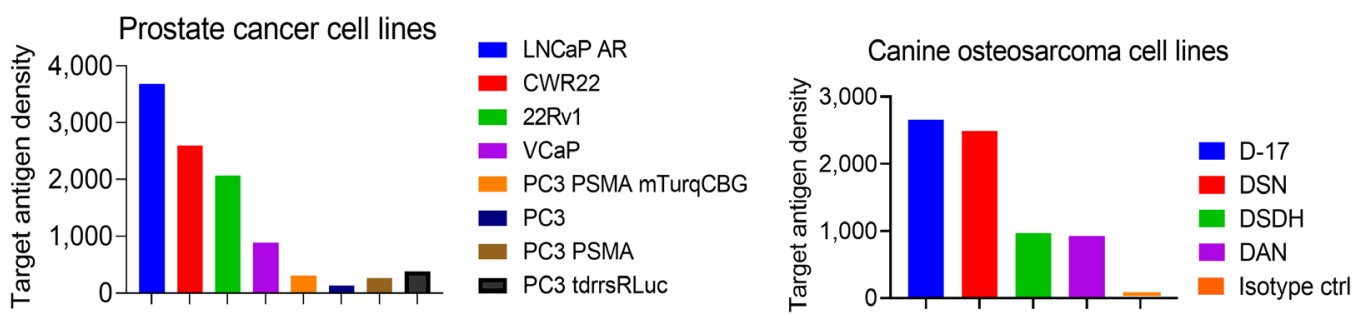

(D)
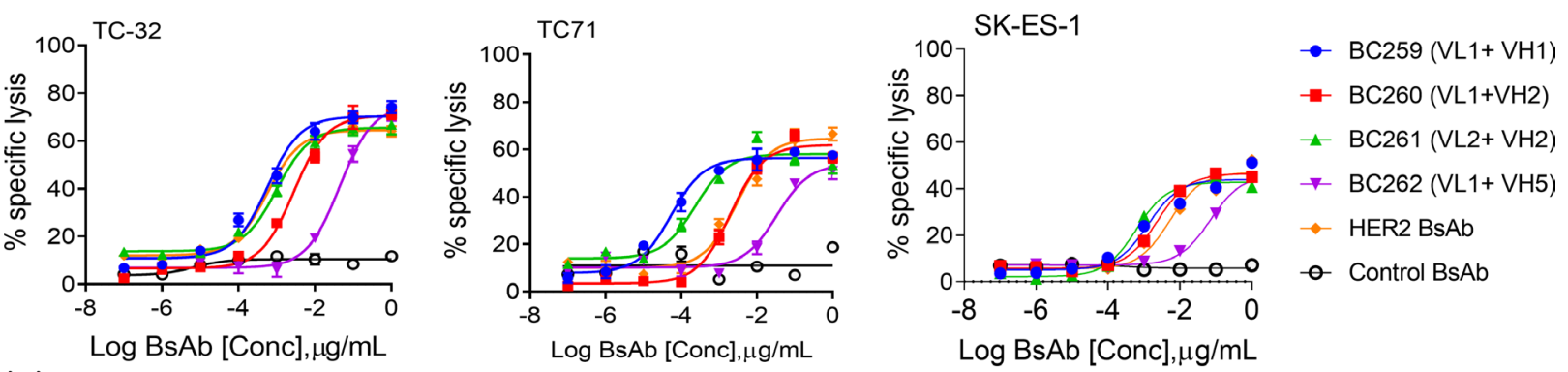

(E)
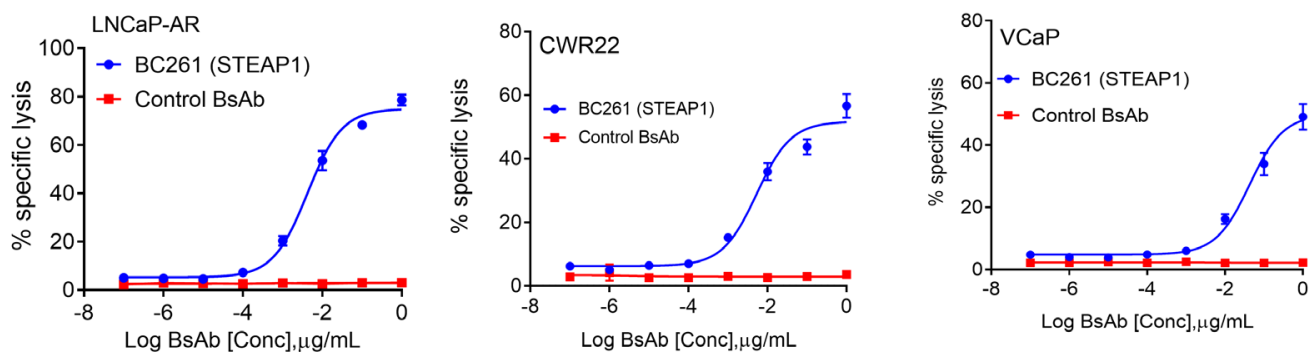

Figure 1 Structure and in vitro antitumor activity of anti-STEAP1 T cell-engaging bispecific antibodies. (A) IgG-[L]-scFv structure of anti-STEAP1 BsAbs. (B) Surface target antigen densities on a panel of Ewing sarcoma family of tumors (EFT) cell lines. EFT cell lines were stained with anti-STEAP1 BsAb BC261, anti-B7-H3 BsAb, anti-GD2 BsAb, or anti-HER2 BsAb, and their binding levels (mean fluorescence intensities (MFIs)) were quantitated using Quantum beads. (C) STEAP1 target antigen density on multiple cancer cell lines including prostate cancer cell lines and canine osteosarcoma cell lines. Cancer cells were stained with BC261, and the MFIs were quantitated using quantum beads. (D) Antibody-dependent T cell-mediated cytotoxicity (ADTC) as a function of increasing doses of anti-STEAP1 BsAbs against EFT cell lines. Effector to target cell ratio (ET ratio) was set to 10:1. (E) In vitro cytotoxicity of BC261 against prostate cancer cell lines. ET ratio was 10:1. CH, constant heavy chain; CL, constant light chain; scFv, single-chain variable fragment; STEAP, six-transmembrane epithelial antigen of prostate; $\mathrm{VH}$, variable heavy chain; VL, variable light chain. 
(A)
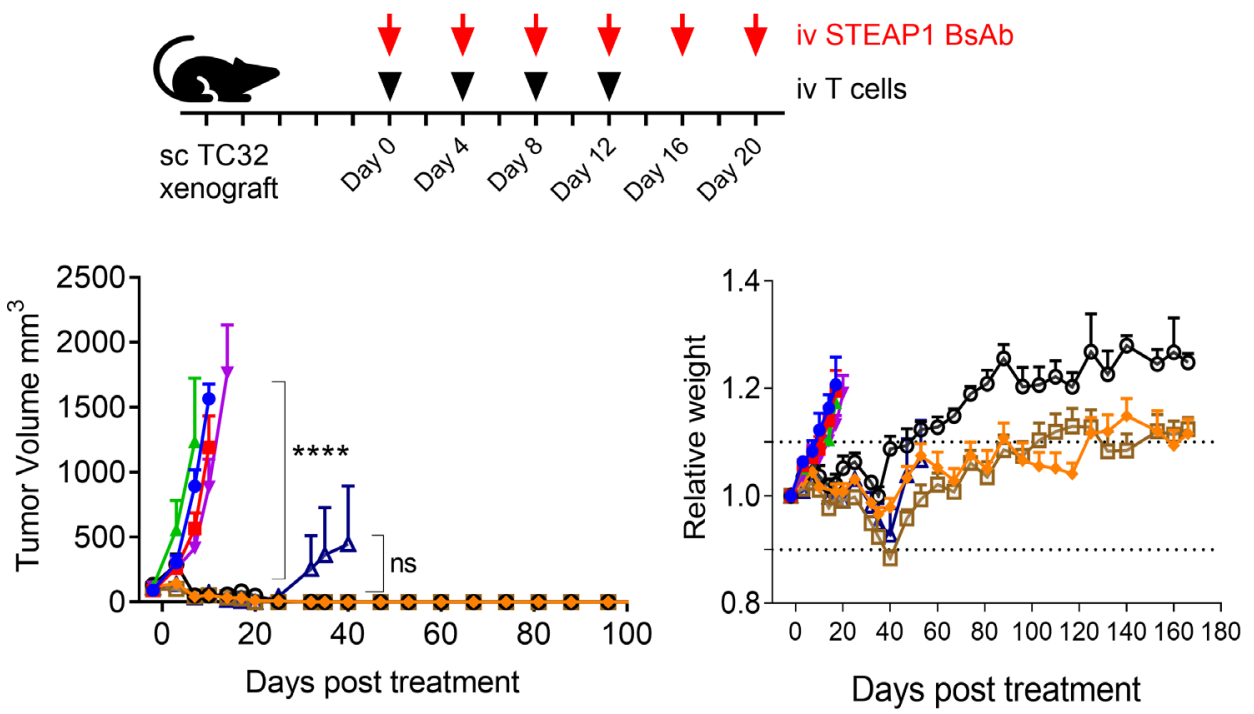

$\rightarrow$ No trearment $(n=5)$

- T cells only $(n=5)$

$\star T$ cells + Control BsAb ( $n=5)$

* T cells + HER2-BsAb ( $n=5)$

$\rightarrow$ T cells + BC259 $(n=5)$

- T cells + BC260 $(n=5)$

廿 T cells $+B C 261(n=5)$

$\triangle$ T cells + BC262 $(n=5)$

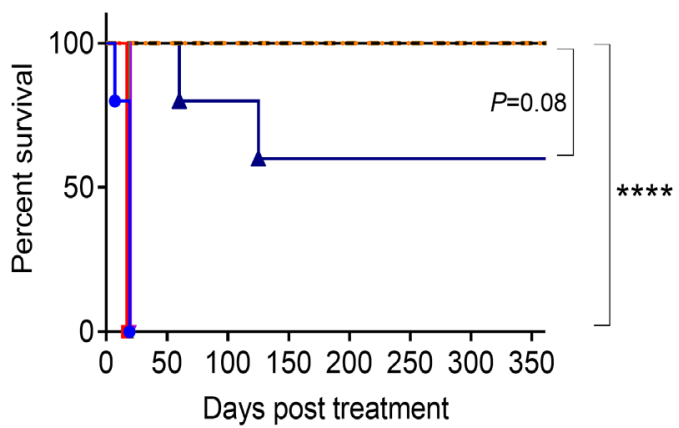

$\rightarrow$ No trearment $(n=5)$

- T cells only $(n=5)$

$\star T$ cells + Control BsAb $(n=5)$

- T cells + HER2-BsAb ( $n=5)$

- T cells + BC260 $(n=5)$

ㄴ. T cells + BC261 $(n=5)$

$\rightarrow$ T cells + BC262 $(n=5)$

- T cells + BC259 $(n=5)$

(B)
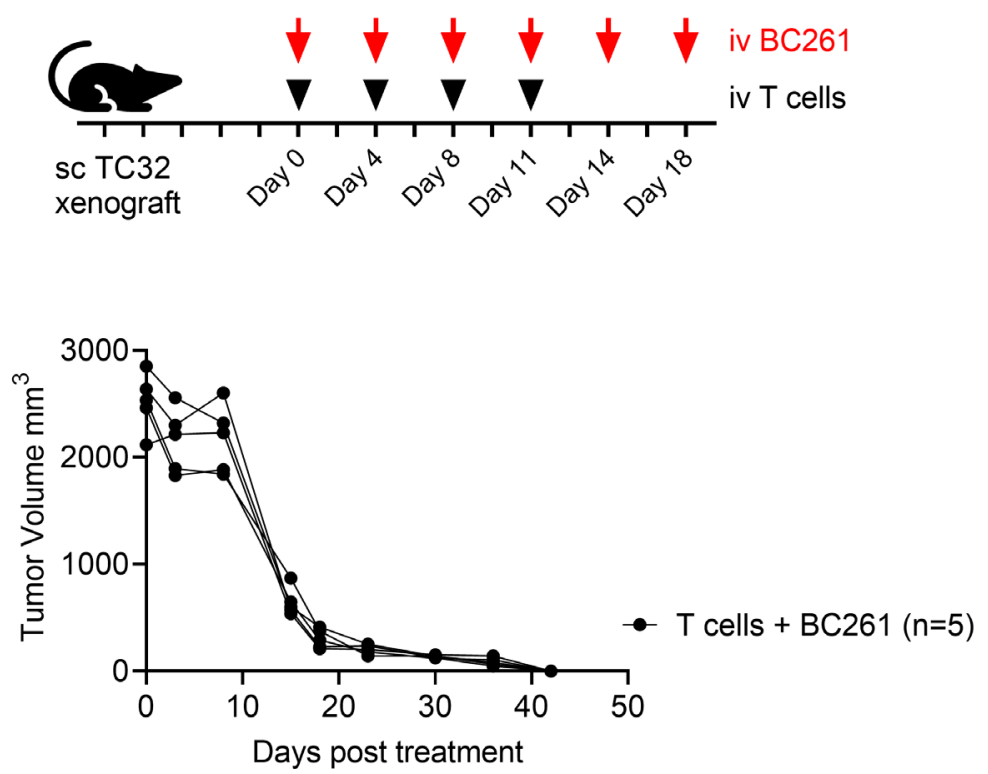

Figure 2 In vivo antitumor activities of STEAP1 bispecific antibodies. (A) In vivo antitumor effect of STEAP1 BsAb in the presence of human T cells against TC-32 cell line xenografts; $3 \times 10^{6}$ cells of TC32 were subcutaneously implanted into mice, and $10 \mu \mathrm{g}$ of STEAP1 BsAb and $2 \times 10^{7}$ of T cells were administered twice per week for $2-3$ weeks to treat the tumors. Tumor growth and relative body weight of mice were monitored, and overall survivals of the mice were plotted. (B) In vivo antitumor effect of BC261 against huge established TC-32 tumor xenografts. When tumors reached $2000 \mathrm{~mm}^{3}, 10 \mu \mathrm{g}$ of BC261 and $2 \times 10^{7}$ of T cells were started. Huge EFT xenografts successfully regressed by BC261 treatment. EFT, Ewing sarcoma family of tumors; STEAP, six-transmembrane epithelial antigen of prostate. 
(A)

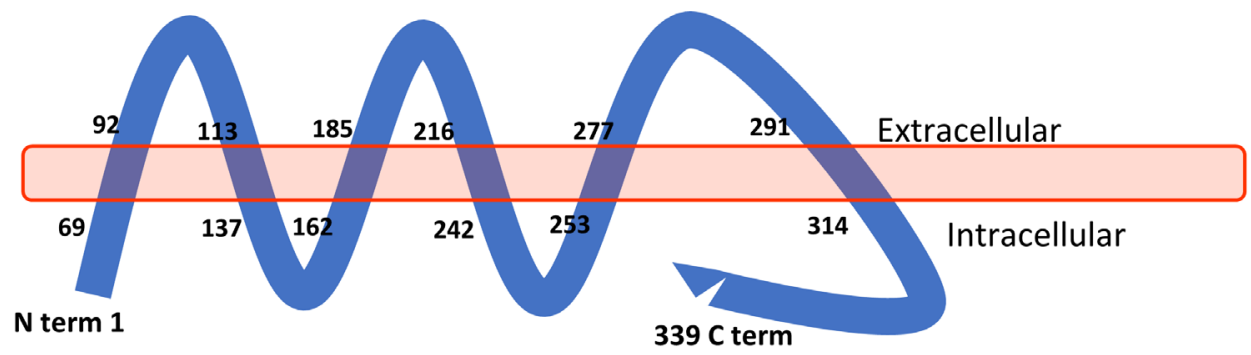

\section{STEAP $1^{\text {st }}$ ECD}

Protein $\backslash$ position

humanSTEAP 1

mouseSTEAP1

caninESTEAP1

\section{STEAP $2^{\text {nd }}$ ECD}

Protein $\backslash$ position

humanSTEAP1

mouseSTEAP1

canineSTEAP1

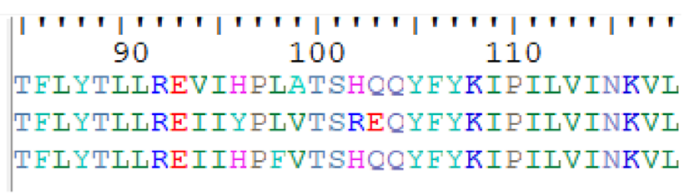

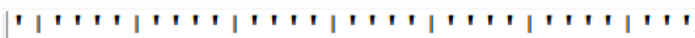
$190200 \quad 210$

MRRSYRYKLLNWAYQQVQQNKEDAW IEHDVWRMEI MRRSYRYKLLNWAYKQVQQNKEDAWVEHDVWRMEI MRRSYRYKLLNWA YQQVQQNKEDAW IEHDVWRMEI

\section{STEAP $3^{\text {rd }}$ ECD}

\section{Protein $\backslash$ position}

humanSTEAP1

mouseSTEAP1

canineSTEAP1

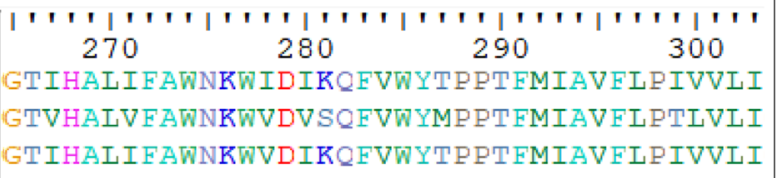

\section{Amino acid differences}

' ', ' ' ' ' ' 100

REVIHPLATSHQQY

..I.Y..V.RE. .

..I...FV.....

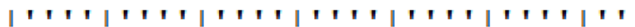
190 200

210

RRSYRYKLLNWAYQQVQQNKEDAWIEHDVWRME

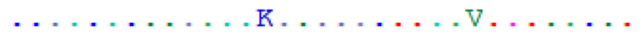
$\ldots \ldots, \ldots+\mu_{n}$

|

WNKWIDIKQFVWYTPPTFMIAV

...v.vS............

$\ldots v \ldots \ldots \ldots \ldots$
(B)

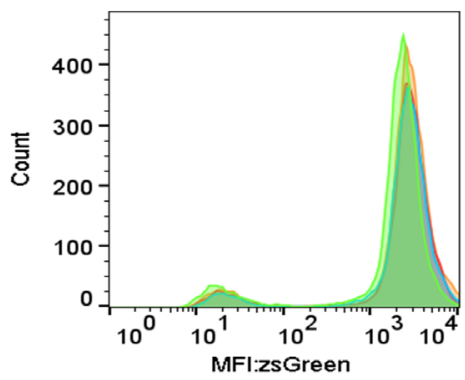

ID

MFI: zsGreen

\begin{tabular}{|c|c|}
\hline STP1h & 1334 \\
\hline STP1m & 1862 \\
\hline STP1mH2 & 1793 \\
\hline STP1mH3 & 1842 \\
\hline
\end{tabular}

(C)

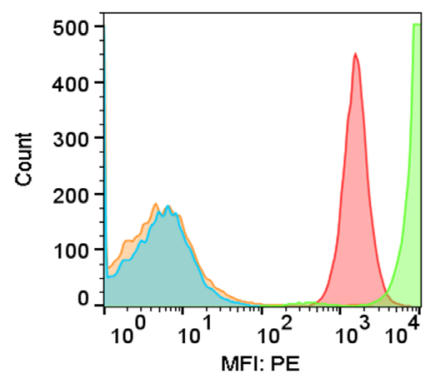

ID

MFI: PE-BC261

\begin{tabular}{|l|r|}
\hline STP1h & 7423 \\
\hline \hline STP1m & 5.00 \\
\hline \hline STP1mH2 & 1415 \\
\hline \hline STP1mH3 & 5.04 \\
\hline
\end{tabular}

Figure 3 Analysis of STEAP1 epitope for STEAP1 bispecific antibodies. (A) Schematic representation of the structural organization of STEAP1 protein in the cell membrane, highlighting the sequence alignment of the extracellular domain (ECD) of human, mouse, and canine STEAP1. (B) Transfection of HEJ293 cells with viral vector carrying human (STP1h), mouse STEAP1 (STP1m), mouse STEAP1 with human second ECD (STP1mH2), or mouse STEAP1 with human third ECD (STP1mH3), tracked by ZsGreen1. (C) Positive staining of STP1mH2 transfectants by STEAP1 BsAb BC261, while negative staining of STP1m or STP1 $\mathrm{mH}$ 2. MFI, mean fluorescence intensity; STEAP, six-transmembrane epithelial antigen of prostate. 
MFI as STP1h, whereas STP1m and STP1mH3 were non-reactive with BC261 (figure 3C), consistent with the location of the epitope on the second ECD of STEAP1. A BLASTp search (https://blast.ncbi.nlm.nih.gov/) confirmed the presence of this second ECD sequence on STEAP1B, encoded by a gene on human chromosome $7 p$ (in contrast to STEAP1 on $7 q$ ). The epitope on the second ECD was also previously reported in two other isoforms, STEAP1B1 and STEAP1B2 in humans ${ }^{47}$ as well as STEAP1 in cynomolgus monkey.

\section{Comparison of antitumor properties of anti-STEAP1 BsAbs built on standard antibody platforms}

Next, we constructed five additional anti-STEAP1 BsAbs (figure 4A) using the identical anti-STEAP1 VH2 and VL2 of BC261, and the anti-CD3 VH and VL of huOKT3. They were named BC328 (STEAP1-CD3 tandem scFv as in $\mathrm{BiTE}^{48}$ ), BC329 (dimeric BiTE using a dimeric $\operatorname{tag}^{49}$ ), BC330 (BiTE-Fc as in half-life extended BiTE ${ }^{50}$ ), BC365 (IgG-[H]-scFv) where the anti-CD3 $\mathrm{scFv}$ is attached to heavy chain instead of light chain, and HD148 (IgG heterodimer) ${ }^{35}$ Cytotoxicity by these alternative $\mathrm{BsAb}$ formats were compared with BC261 (IgG-[L]-scFv) in vitro and in vivo. All these BsAbs showed high purity $(>90 \%)$ by HPLC and high stability after freeze and thaw cycles or heat stress at $40^{\circ} \mathrm{C}$ for more than 2 weeks (online supplemental table 7). When we compared in vitro potency among STEAP1 BsAb formats, BiTE-Fc and IgG heterodimer were the least effective in killing tumor cells, contrasting with comparable in vitro cytotoxicity by the other four BsAbs (figure 4B).

Given the different BsAb protein size, plasma pharmacokinetics could confound direct comparison of in vivo potency among BsAbs. We chose to deliver these BsAbs prebound to $\mathrm{T}$ cells using a method previously described. ${ }^{51}$ Luciferase transduced $\mathrm{T}$ cells $(\mathrm{Luc}(+) \mathrm{T}$ cells) were armed with each of the six different formats of anti-STEAP1 BsAb and were injected intravenously into EFT PDX (ES15a) bearing mice, and tumor-infiltrating lymphocytes (TILs) was monitored over time by bioluminescence imaging (figure 4C). Although all 6 BsAbs successfully drove T cells into tumors, BC261 (IgG-[L]$\mathrm{scFv}$ ) armed $\mathrm{T}$ cells showed the most robust TIL infiltration based on BLIs, with tens of times higher AUC for IgG-[L]-scFv when compared with other formats (online supplemental table 8 ). The peak BLI for IgG-[L]-scFv was $>100$-fold higher than those for other BsAb armed $\mathrm{T}$ cells, with the most persistence $(p<0.0001)$. The BLI findings were confirmed by the CD3 IHC staining of tumors showing abundant $\mathrm{T}$ cell infiltration with BC261 armed T cells (figure 4D).

This superiority of BC261 in increasing TILs and their in vivo persistence translated into substantial and significant differences in antitumor response against EFT PDXs. STEAP1 BsAb armed T cells $\left(10 \mu \mathrm{g}\right.$ of BsAb $/ 2 \times 10^{7}$ cells) were administered intravenously on day 0 and day 14 (figure 5A). BC261 (IgG-[L]-scFv) armed T cells were the most effective without overt toxicities (figure 5B).
This superior potency of the IgG-[L]-scFv platformed BC261 was confirmed in another EFT PDX (ES3a) mouse model (figure 5C). Here, two doses STEAP1 $\mathrm{BsAb}$ armed $\mathrm{T}$ cells were administered on day 0 and day 4. BC261 armed T cells induced complete remission of established tumors and significantly prolonged survival $(p<0.0001)$, contrasting with other formats of STEAP1 $\mathrm{BsAb}$ armed $\mathrm{T}$ cells (figure 5D). The mice treated with BC261 retained their body weight, with no clinical abnormalities throughout the observation period. Among them treated with BC261 armed T cells three mice showed tumor relapse between day 60 and day 90 post-treatment. Interestingly, the relapsed tumors which regrew to 200$2000 \mathrm{~mm}^{3}$ in size responded again to retreatment with two injections of BC261 armed T cells (on day 102 and day 106), and the remission was durable past 200 days (data not shown).

In vivo cytokine release was also compared among six different formats of anti-STEAP1 BsAb. Serum TH1 cell cytokine levels were measured at different time points after STEAP1-EAT injection in EFT PDX (ES3a) xenografted mice (online supplemental figure 4A). There was no significant difference in the levels of IL-6, IL-10, and TNF- $\alpha$ among the six different BsAb platforms. When analyzed by AUC of cytokine levels over time (online supplemental figure 4B), only IL-2 and IFN- $\gamma$ levels differed among six groups of STEAP1 BsAb $(\mathrm{p}=0.0016$, and $\mathrm{p}=0.0027$, respectively), with BC261 (IgG-[L]-scFv) showing the highest levels of IL-2 and IFN- $\gamma$.

\section{Dose response of $\mathrm{BC261}$}

We titrated the dose of BC261 required for sufficient antitumor effects in vivo. Tumor control was achieved over a range of BsAb doses from $2 \mu \mathrm{g}$ to $50 \mu \mathrm{g}$ per dose without toxicities, surpassing the antitumor effect of HER2-BsAb (online supplemental figure 5). For durable remission and long-term survival, $10 \mu \mathrm{g} /$ dose of BC261 was chosen for the rest of the in vivo experiments.

\section{Anti-tumor effect of BC261 against a panel of EFT xenografts} Antitumor activity of BC261 was tested in multiple EFT xenograft mouse models (figure 6A). While GD2-BsAb failed to suppress TC71 CDX tumor growth, BC261 successfully ablated tumors and improved survival without obvious toxicities (figure 6B). In another EFT cell line SK-ES-1 CDX model, BC261 exerted potent in vivo antitumor effect again, successfully prolonging survival (figure 6C). EFT PDX models, ES03a and ES15a, were also treated with intravenous BC261 and T cells, and durable remission was achieved with BC261 (online supplemental figure 6). Although both PDX tumors were GD2(+) and HER2(+) by immunohistochemical staining, GD2-BsAb was substantially less effective than BC261, whereas HER2-BsAb was comparable to BC261.

\section{In vivo antitumor response of BC261 against prostate cancer}

In addition, since STEAP1 is present on prostate cancer cells (figure 1C), antitumor efficacy of BC261 was evaluated 
(A)

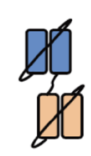

Monomeric BiTE BC328

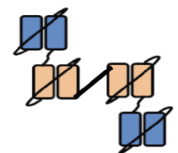

Dimeric BiTE BC329

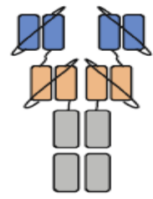

BiTE-FC

BC330

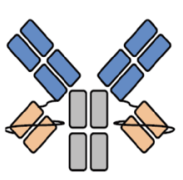

IgG-[L]-scFv BC261

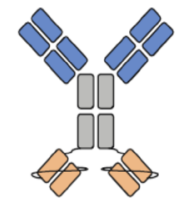

$\operatorname{lgG}-[\mathrm{H}]-\mathrm{SCFV}$ BC365

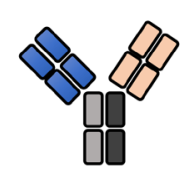

IgG Heterodimer HD148

(B)
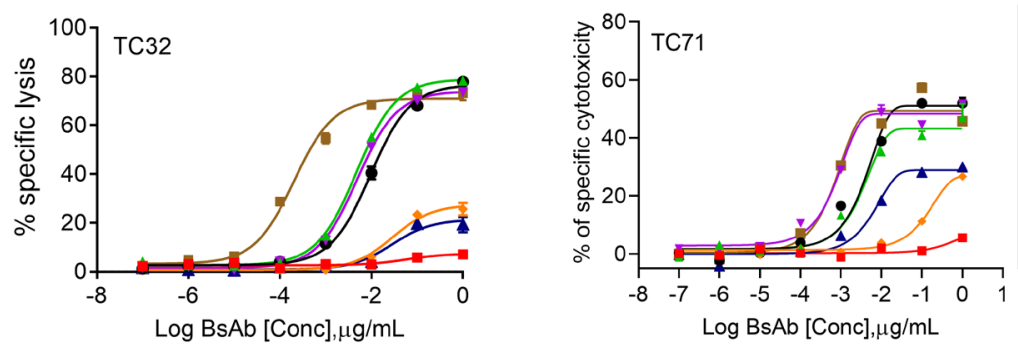

(C)

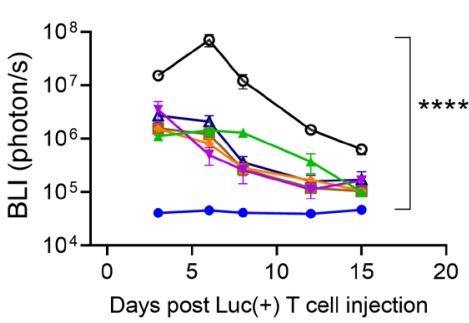

- Control BsAb (GPA33)

- BC328 (monomeric BiTE)

- BC329 (dimeric BiTE)

$\rightarrow$ BC330 (BiTE-FC)

- BC261 (IgG-[L]-scFv)

- BC365 (IgG-[H]-scFv)

- HD148 (IgG heterodimer)

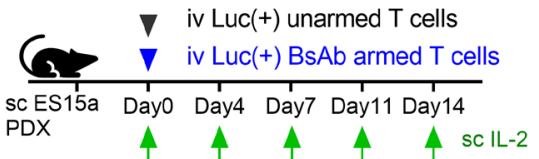

Days post Luc(+) T cell injection

- No treatment $(n=5)$

$\rightarrow$ BC328 (BiTE) $(n=5)$

* BC329 (dimeric BiTE) $(n=5)$

$\rightarrow$ BC330 (BiTE-Fc) $(n=5)$

- BC261 (IgG-[L]-scFv) (BC261) (n=5)

- BC365 (lgG-[H]-scFv) ( $n=5)$

$\triangle$ HD148 (lgG heterodimer) $(n=5)$

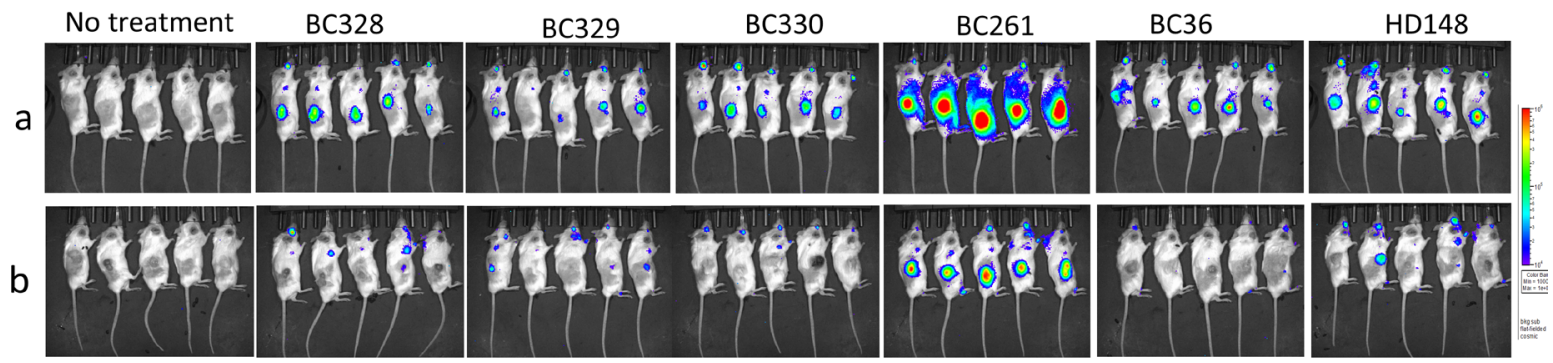

(D)
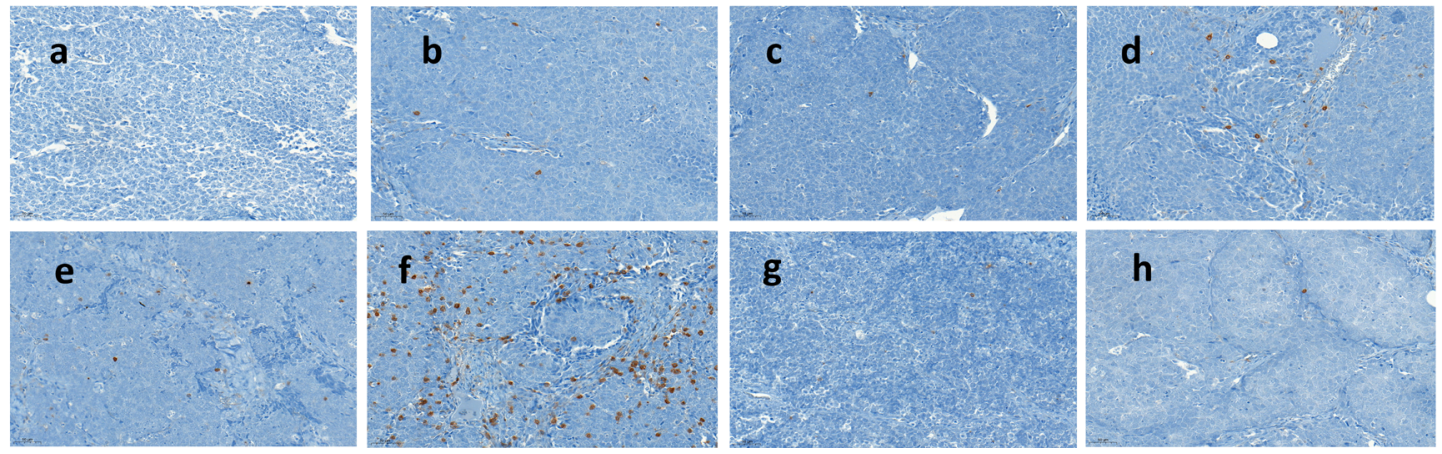

Figure 4 Antitumor activities of anti-STEAP1 T cell-engaging bispecific antibody. (A) Schematic representation of the six structural formats of STEAP1 BsAb. ${ }^{35}$ (B) Antibody-dependent T cell-mediated cytotoxicity (ADTC) assay against EFT cell lines (Tc32 ad TC71) using different formats of STEAP1 BsAb. Effector to target cell ratio (ET ratio) was 10:1. (C) Bioluminescence imaging (BLI) of Luc(+) T cells armed with six different formats of STEAP1 BsAb on (a) day 6 and (b) day 18 post treatment and quantitation of bioluminescence intensity in the lesions of tumor. Luciferase transduced T cells (Luc(+) T cells) were armed with various formats of STEAP1 BsAb (10 $\mathrm{gg}$ of STEAP1 BsAb/2 $\times 10^{7}$ of T cells) and administered with supplementary IL-2 (1000) IU/ dose) to the mice bearing EFT PDXs (ES15a), and the BLIs were followed. (D) TC32 EFT cell line xenografts were harvested on

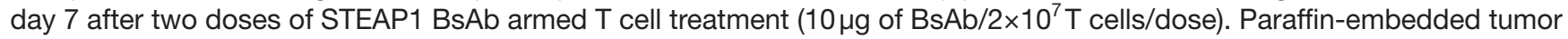
sections were stained with CD3 (x10): a, no treatment; b, control BsAb; c, BC328; d, BC329; e, BC330; f, BC261; g, BC365; h, HD148. EFT, Ewing sarcoma family of tumors; PDXs, patient-derived tumor xenografts; STEAP, six-transmembrane epithelial antigen of prostate. 
(A)

(B)

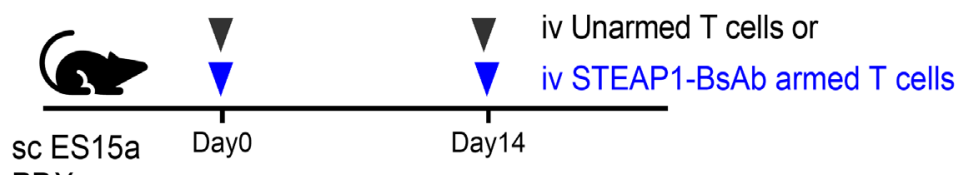

PDX
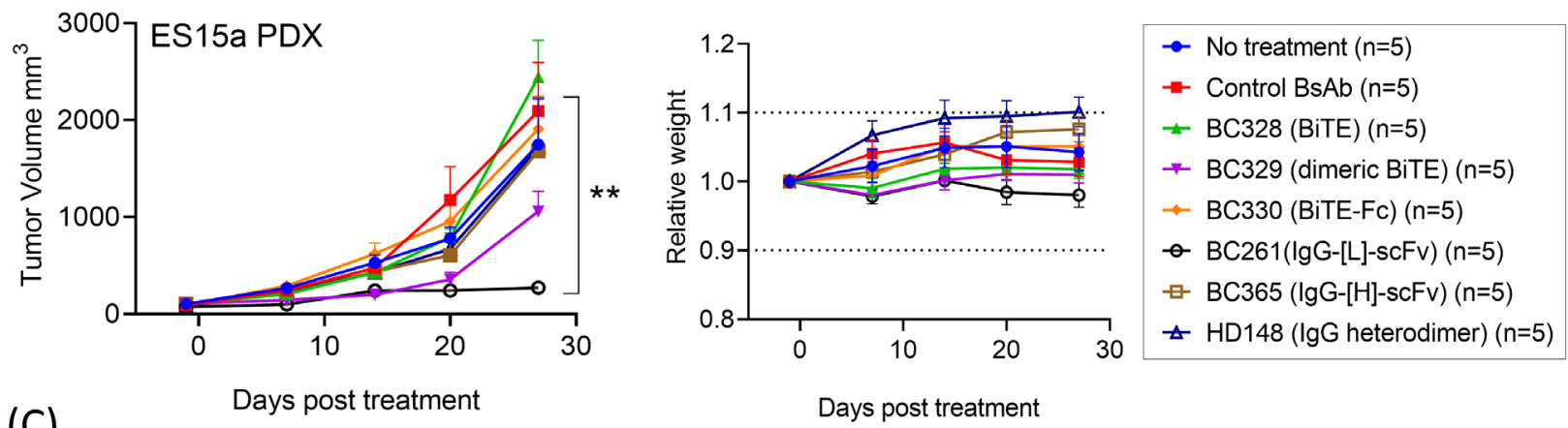

(C)

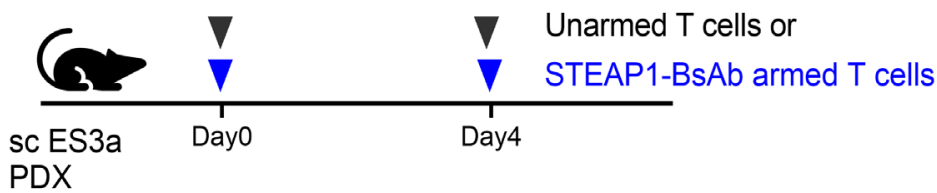

(D)
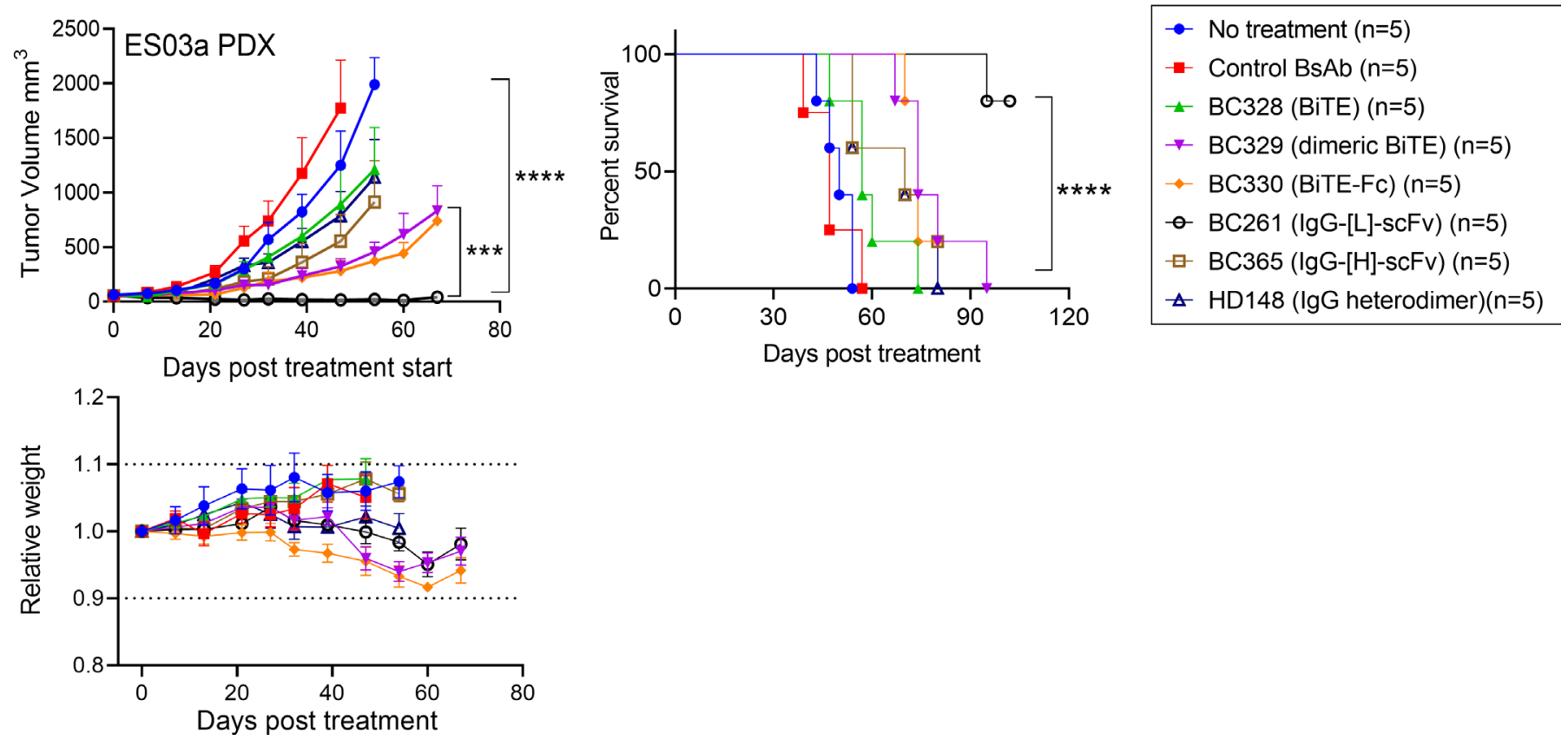

Figure 5 In vivo antitumor effect of STEAP1 bispecific armed T cells. (A) T cells armed with six different formats of STEAP1

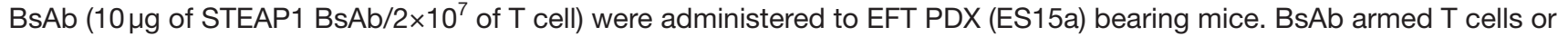
unarmed T cells were administered on day 0 and day 14, and subcutaneous (SC) IL-2 (1000 IU) was supplemented twice per week. (B) In vivo antitumor effect of six different formats of STEAP1 BsAb armed T cells against EFT PDXs (ES15a) and relative body weight of mice after treatment. (C) T cells were armed with six different formats of STEAP1 BsAb (10 $\mu \mathrm{g}$ of STEAP1 $\mathrm{BsAb} / 2 \times 10^{7}$ of T cell) and tested for in vivo efficacy against different EFT PDXs (ES3a). BsAb armed T cells or unarmed T cells were administered on day 0 and day 4, and SC IL-2 was supplemented twice per week. (D) In vivo antitumor effect of six different formats of STEAP1 BsAb armed T cells against EFT PDX (ES3a) and relative body weight of mice after treatment. EFT, Ewing sarcoma family of tumors; PDXs, patient-derived tumor xenografts; STEAP, six-transmembrane epithelial antigen of prostate. 
(A)

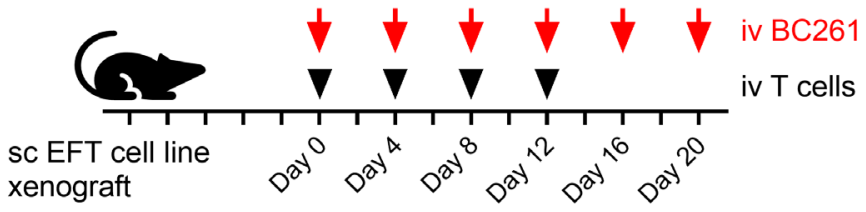

(B)
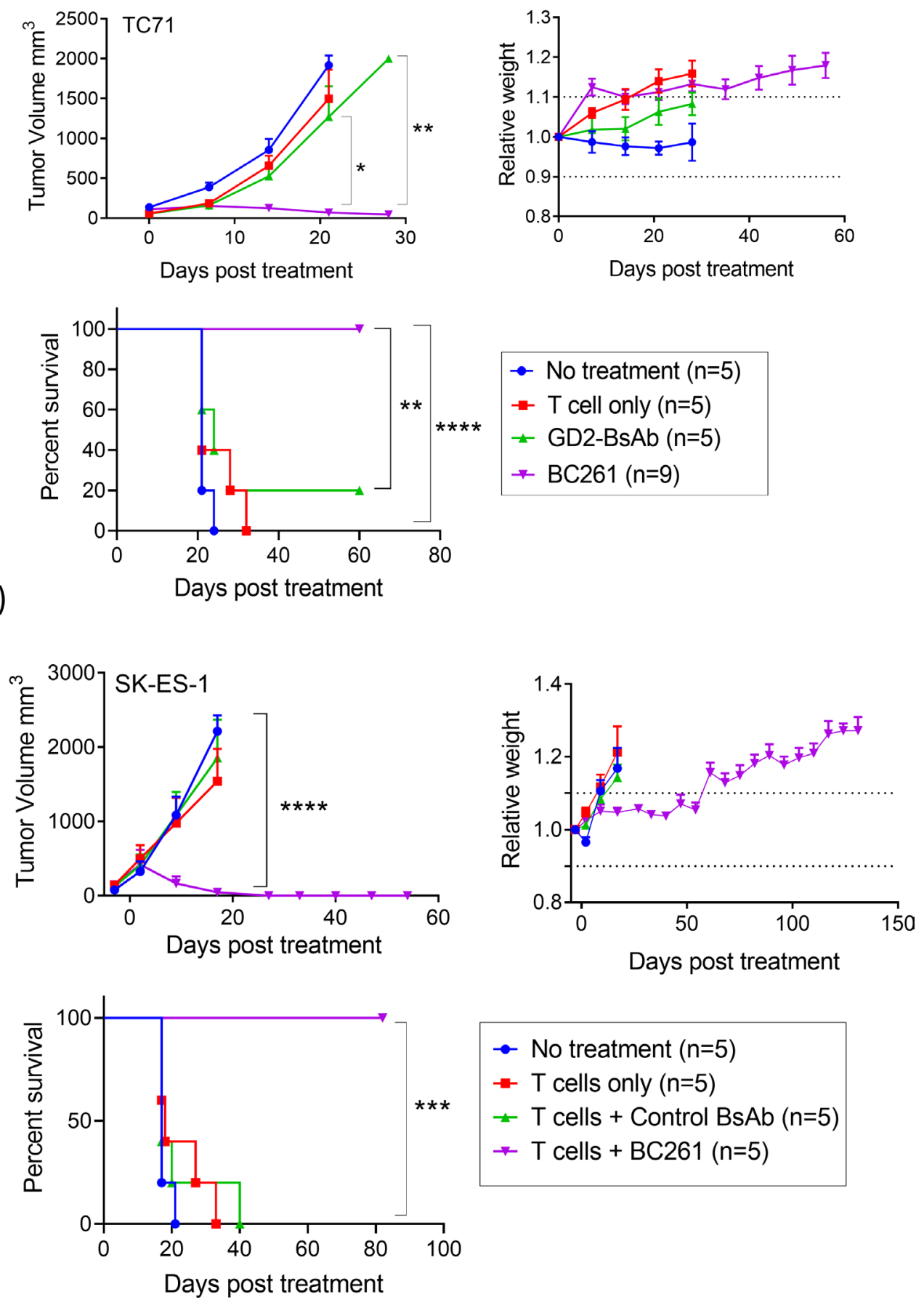

Figure 6 In vivo antitumor effect of BC261 against a panel of STEAP1(+) EFT cell line xenografts (CDXs). (A) Ten micrograms of BC261 was administered with $2 \times 10^{7}$ of T cells twice per week for $2-3$ weeks to treat EFT cell line xenografts. Subcutaneous IL-2 (1000 IU) was supplemented with each T cell injection. (B) In vivo antitumor effect of BC261 against EFT TC-71 cell line xenografts. Tumor growth and relative body weight of mice were monitored, and overall survivals were plotted. (C) In vivo antitumor effect of BC261 against EFT SK-ES-1 cell line xenografts. Tumor growth and relative body weight of mice were monitored, and overall survivals were plotted. EFT, Ewing sarcoma family of tumors; STEAP, six-transmembrane epithelial antigen of prostate. 
against two prostate cancer PDXs (figure 7A). Despite the rapid tumor growth to $>500-1000 \mathrm{~mm}^{3}$ size over 1 week, BC261 successfully ablated all TM00298 PDX tumors, significantly prolonging survival $(\mathrm{p}=0.0002)$ (figure $7 \mathrm{~B}$ ). In a second prostate cancer PDX model, J000077451, BC261 again demonstrated potent antitumor efficacy (figure 7C). Mice treated with the BC261 elicited potent tumor regression and prolonged survival without clinical toxicity.

\section{DISCUSSION}

This report describes the first discovery and validation of a novel anti-STEAP1 T-BsAb BC261 built on the IgG-[L]scFv platform with exceptional in vivo antitumor potency. The epitope of STEAP1 BsAb was mapped to the second ECD of STEAP1, an epitope shared among human, canine and cynomolgus monkeys. The BC261 was effective against large established xenografts derived from EFT and prostate cancers despite their modest levels of STEAP1 expression. BC261 showed exceptional antitumor efficacy compared with standard T-BsAb platforms in driving $\mathrm{T}$ cells into tumors and suppressing tumor growth, enabling long-term disease-free survival.

Although the superiority of the 'IgG-[L]-scFv' platform for building T-BsAb has been previously reported for disialogangliosides (GD2), ${ }^{35} 36$ a carbohydrate antigen anchored in the membrane by a short lipid side chain, the superior potency of this format has never been verified for protein targets. This is the first report to demonstrate this functional superiority of the IgG-[L]-scFv platform for the protein target STEAP1. We compared antitumor effects of representative BsAb formats and confirmed the exceptional antitumor efficacy of STEAP1 BsAb BC261 both as standalone therapeutics and when used for arming $\mathrm{T}$ cells targeting a broad spectrum of human cancers.

For T-BsAb, protein size, valency, affinities, and structure can influence their biodistribution, ${ }^{52}$ clearance, and potency. ${ }^{53}$ STEAP1 BsAb BC261 was developed using the IgG-[L]-scFv BsAb platform which has longer half-life with the larger protein size $(\sim 210 \mathrm{KD})^{54}$ and increased CD3 T cell affinity with two anti-CD3 domains. This T-BsAb format maintains bivalency toward the tumor target to maintain avidity, with functional monovalency of CD3 engagement through affinity reduction by mutating cysteine to serine in one of the CDRs. In this format, neonatal Fc receptor $[\mathrm{FcR}(\mathrm{n})]$ and protein $\mathrm{A}$ binding are retained, while mutations N297A and K322A are introduced to silence Fc functions, thereby reducing complement-mediated cytotoxicity or antibody-dependent cell-mediated cytotoxicity. ${ }^{44} \mathrm{~T}$-BsAbs built using this IgG-[L]-scFv format against GD2, HER2, GPA33, CD33, CD1932 $394655-57$ and now against STEAP1 have succeeded in driving polyclonal $\mathrm{T}$ cells into human tumor xenografts rapidly and quantitatively, producing robust antitumor responses within 2-3 weeks after treatment start, which did not overlap temporally with the xenogeneic GVHD effect which developed between 4 weeks and 8 weeks post-treatment.
The exceptional potency of BC261 could partly be attributed to the properties of target antigen STEAP1. STEAP1 is highly expressed in EFT, and its expression correlates with the EWS-ETS fusion proteins. ${ }^{58}{ }^{59}$ STEAP1 is induced by EWS-FLII translocation, and its expression promotes proliferation, invasiveness, anchorageindependent colony formation, tumorigenicity, and metastasis of EFT, ${ }^{59}$ implicating close relationship between STEAP1 and the EWS-ETS translocation. Interestingly, all relapsed tumors after BC261 therapy showed regression when retreated with BC261-EATs (online supplemental figure 5). This sensitivity to retreatment contrasts with most published studies where tumor escape following $\mathrm{T}$ cell immunotherapy is typically associated with target antigen loss or downregulation. ${ }^{4160}$ If STEAP1 expression is associated with cancer cell stemness, a property driven by the EWS-ETS fusion protein, it could explain the high potency of BC261 targeting STEAP1. ${ }^{61}$ It is also notable that $50 \%-70 \%$ of prostate cancers have similar chromosomal rearrangements resulting in aberrant expression of an oncogenic ETS family transcription factor, and these ETS factors interact with the Ewing sarcoma breakpoint protein EWS, leading to gene activation, cell migration, transformation, and tumorigenesis. ${ }^{62}$ Understanding the mechanistic pathways and biology behind STEAP1 and EWS/ETS-related fusions should help future design and testing of immunotherapeutics directed at STEAP1. ${ }^{3}$

One of the major findings of our study is the discovery of the shared epitope of STEAP1 among human, dogs, and non-human primates (NHP). 'On-target off-tumor toxicities' from cellular immunotherapy can induce serious side effects that are difficult to predict in current preclinical mouse models due to the specificity of BsAbs or CARs for human antigens. Although STEAP1 expression is highly restricted to tumor tissues and absent in vital organs, it is found at lower levels in normal tissues such as prostate, bladder, liver, kidney, pancreas, and skeletal muscle. ${ }^{2027} 63$ As a non-human preclinical model, dogs and NHP may provide alternatives for evaluating not only the efficacy but also the 'on-target off tumor toxicity'. Besides, canine osteosarcoma is one of the most common cancers in dogs. ${ }^{64}$ As an outbred nonhuman tumor model, it provides an alternative for testing STEAP1 antibody-based therapeutics both for toxicities and for efficacy. Since osteosarcoma is also difficult to cure in canine hosts, STEAP1-targeted antibody-based therapeutics could fulfill another unmet canine need. Even though the STEAP1 protein is highly restricted to tumors, long-term persistence of the cytotoxic drugs or cytotoxic immune effector cells could be harmful. While CAR $T$ cells are life-long and such toxicities could be prolonged and life-threatening, BsAb-driven $\mathrm{T}$ cells or EATs have limited functional life expectancy only weeks. As the BsAbs get metabolized, $T$ cells should revert to their non-specific states, reducing or terminating the risk of life-threatening long-term toxicities. In this respect, targeting STEAP1 using T-BsAb and EAT offers safter alternatives. 
(A)

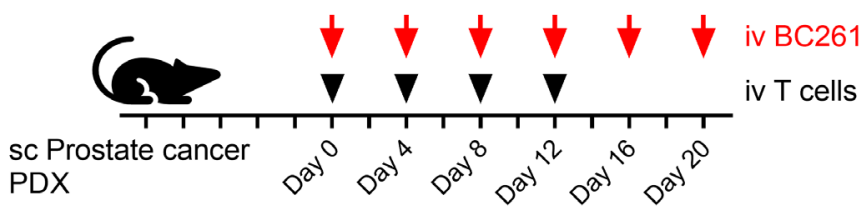

(B)
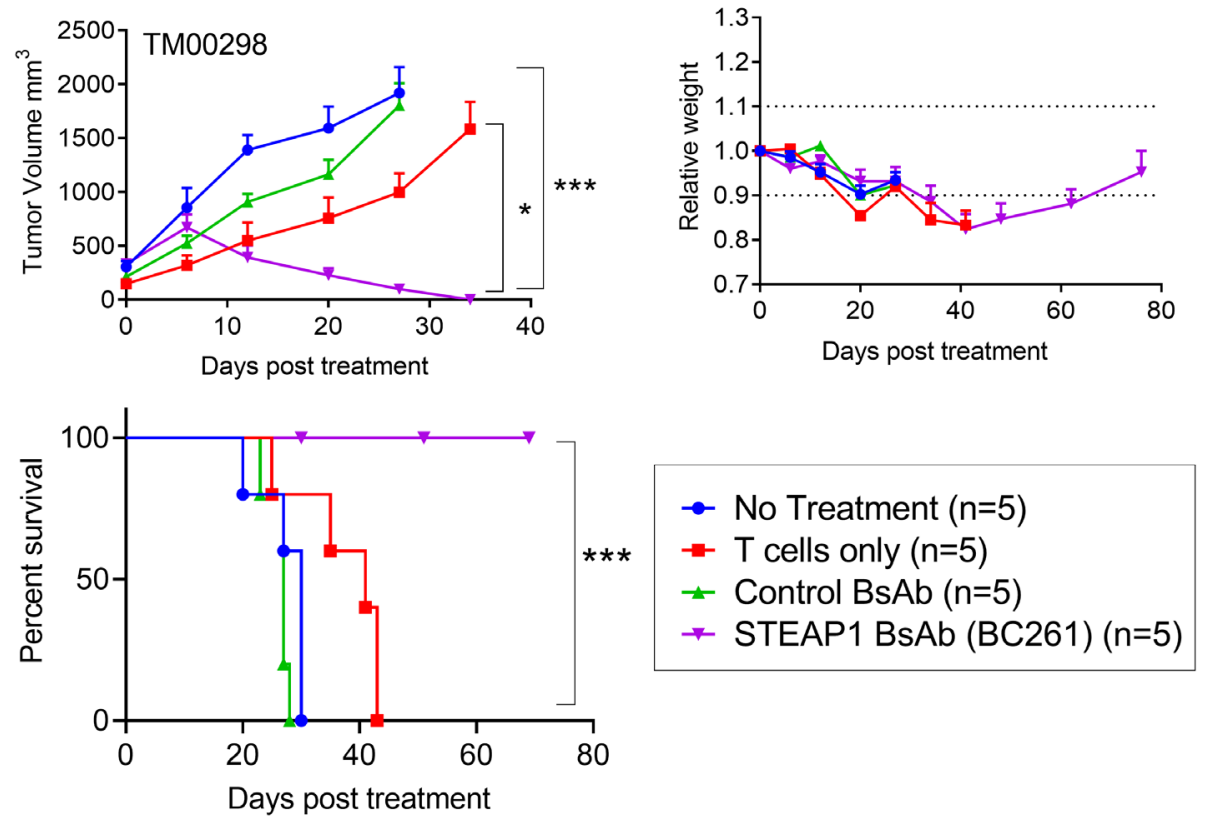

(C)
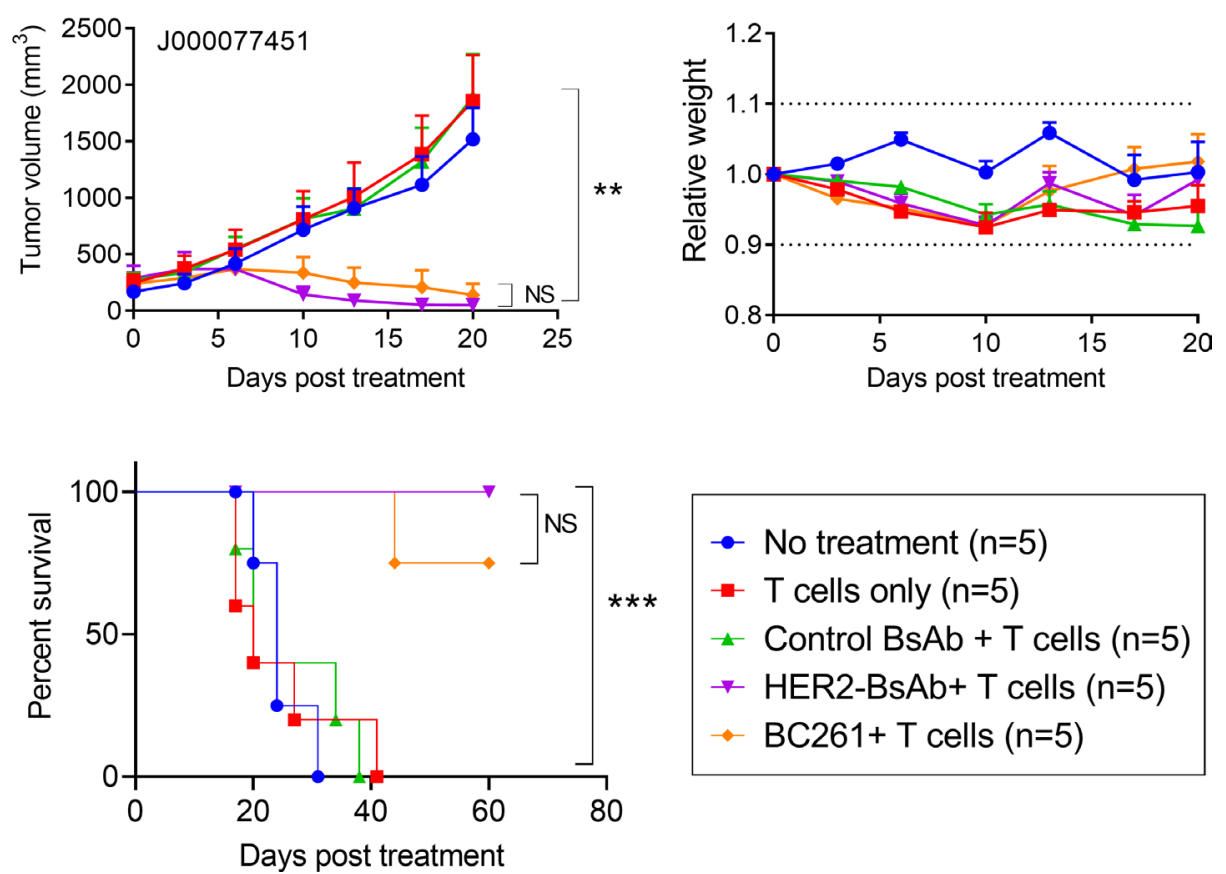

$$
\begin{aligned}
& - \text { No treatment }(n=5) \\
& - \text { T cells only }(n=5) \\
& - \text { Control BsAb + T cells }(n=5) \\
& \rightarrow \text { HER2-BsAb+T cells }(n=5) \\
& \rightarrow \text { BC261+T cells }(n=5)
\end{aligned}
$$

Figure 7 In vivo antitumor effect of BC261 against STEAP1(+) prostate cancers. (A) Ten micrograms of BC261 was administered with $2 \times 10^{7}$ of T cells twice per week for 2-3 weeks to treat prostate cancer PDXs. Subcutaneous IL-2 (1000 IU) was supplemented with each T cell injection. (B) In vivo antitumor effect of BC261 against prostate cancer PDX TM00298. (C) In vivo antitumor effect of BC261 against prostate cancer PDX J000077451. Tumor growth, relative body weight, and overall survival after treatment were plotted and compared. PDXs, patient-derived tumor xenografts; STEAP, six-transmembrane epithelial antigen of prostate. 
Taken together, STEAP1 is an appealing target for T cell immunotherapy, and STEAP1 T-BsAb BC261 offers a novel solution to engage $\mathrm{T}$ cells to traffic into immunologically 'cold' STEAP1 $(+)$ malignancies. BC261 induced robust antitumor responses without long-term toxicities for stand-alone BsAb or BsAb armed T cells, warranting clinical development for human solid tumors with major unmet need, such as EFT and prostate cancers.

\section{Twitter Tsung-Yi Lin @StevenTYLin08}

Acknowledgements We would like to especially thank Xu Hong for analyzing STEAP1 BsAbs' affinity to CD3.

Contributors T-YL, JAP, and N-KVC designed the experiments, interpreted and analyzed the results, and wrote the manuscript. JAP and N-KVC revised the manuscript. AL did experiments with the prostate cancer PDX mouse model. $\mathrm{H}-\mathrm{FG}$ tested the purity and stability of the STEAP1-BsAbs using HPLC and SDS-PAGE and analyzed STEAP1 antigen expression on multiple cancer cell lines. All authors reviewed this manuscript and approved the final manuscript.

Funding This work was partly supported by Enid A. Haupt Endowed Chair, the Robert Steel Foundation, and Kids Walk for Kids with Cancer. Technical service provided by the MSK Animal Imaging Core Facility, Antitumor Assessment Core Facility, and Molecular Cytology Core Facility were supported in part by the $\mathrm{NCl}$ Cancer Center Support Grant P30 CA008748.

Competing interests Both MSK and NKC have financial interest in Y-mAbs, Abpro-Labs and Eureka Therapeutics. NKC reports receiving commercial research grants from Y-mAbs Therapeutics and Abpro-Labs Inc. NKC was named as inventor on multiple patents filed by MSK, including those licensed to Ymabs Therapeutics, Biotec Pharmacon, and Abpro-labs. NKC is a SAB member for Abpro-Labs and Eureka Therapeutics.

Patient consent for publication Not required.

Ethics approval All animal experiments were approved by the Memorial Sloan Kettering's Institutional Animal Care and Use Committee (IACUC) and were executed according to the ACUC guidelines. Patient-derived tumor xenografts were established with MSKCC IRB approval.

Provenance and peer review Not commissioned; externally peer reviewed.

Data availability statement All data relevant to the study are included in the article or uploaded as supplementary information. All data generated or analyzed during this study are included in this published article.

Supplemental material This content has been supplied by the author(s). It has not been vetted by BMJ Publishing Group Limited (BMJ) and may not have been peer-reviewed. Any opinions or recommendations discussed are solely those of the author(s) and are not endorsed by BMJ. BMJ disclaims all liability and responsibility arising from any reliance placed on the content. Where the content includes any translated material, BMJ does not warrant the accuracy and reliability of the translations (including but not limited to local regulations, clinical guidelines, terminology, drug names and drug dosages), and is not responsible for any error and/or omissions arising from translation and adaptation or otherwise.

Open access This is an open access article distributed in accordance with the Creative Commons Attribution Non Commercial (CC BY-NC 4.0) license, which permits others to distribute, remix, adapt, build upon this work non-commercially, and license their derivative works on different terms, provided the original work is properly cited, appropriate credit is given, any changes made indicated, and the use is non-commercial. See http://creativecommons.org/licenses/by-nc/4.0/.

\section{ORCID iDs}

Jeong A Park http://orcid.org/0000-0003-3690-6747

Nai-Kong V Cheung http://orcid.org/0000-0001-6323-5171

\section{REFERENCES}

1 Esiashvili N, Goodman M, Marcus RB. Changes in incidence and survival of Ewing sarcoma patients over the past 3 decades: surveillance epidemiology and end results data. J Pediatr Hematol Oncol 2008;30:425-30.

2 Schmiedel BJ, Hutter C, Hesse M, et al. Expression of multiple membrane-associated phospholipase A1 beta transcript variants and lysophosphatidic acid receptors in Ewing tumor cells. Mol Biol Rep 2011;38:4619-28.

3 Arvand A, Denny CT. Biology of EWS/ETS fusions in Ewing's family tumors. Oncogene 2001;20:5747-54.

4 May WA, Gishizky ML, Lessnick SL, et al. Ewing sarcoma 11;22 translocation produces a chimeric transcription factor that requires the DNA-binding domain encoded by FLI1 for transformation. Proc Natl Acad Sci U S A 1993;90:5752-6.

5 Janknecht R. Ews-Ets oncoproteins: the linchpins of Ewing tumors. Gene 2005;363:1-14.

6 Grier HE, Krailo MD, Tarbell NJ, et al. Addition of ifosfamide and etoposide to standard chemotherapy for Ewing's sarcoma and primitive neuroectodermal tumor of bone. $N$ Engl $J$ Med 2003;348:694-701.

7 Cotterill SJ, Ahrens S, Paulussen M, et al. Prognostic factors in Ewing's tumor of bone: analysis of 975 patients from the European intergroup cooperative Ewing's sarcoma Study Group. J Clin Oncol 2000;18:3108-14.

8 Kopp LM, Katsanis E. Targeted immunotherapy for pediatric solid tumors. Oncoimmunology 2016;5:e1087637.

9 Wedekind MF, Denton NL, Chen C-Y, et al. Pediatric cancer immunotherapy: opportunities and challenges. Paediatr Drugs 2018;20:395-408.

10 Berghuis D, de Hooge ASK, Santos SJ, et al. Reduced human leukocyte antigen expression in advanced-stage Ewing sarcoma: implications for immune recognition. J Pathol 2009;218:222-31.

11 Evans $\mathrm{CH}$, Liu F, Porter RM, et al. EWS-FLI-1-targeted cytotoxic T-cell killing of multiple tumor types belonging to the Ewing sarcoma family of tumors. Clin Cancer Res 2012;18:5341-51.

12 Foell JL, Hesse M, Volkmer I, et al. Membrane-Associated phospholipase A1 beta (LIPI) is an Ewing tumour-associated cancer/ testis antigen. Pediatr Blood Cancer 2008;51:228-34.

13 Liu XF, Helman LJ, Yeung C, et al. XAGE-1, a new gene that is frequently expressed in Ewing's sarcoma. Cancer Res 2000;60:4752-5.

14 Krishnadas DK, Shusterman S, Bai F, et al. A phase I trial combining decitabine/dendritic cell vaccine targeting MAGE-A1, MAGE-A3 and NY-ESO-1 for children with relapsed or therapy-refractory neuroblastoma and sarcoma. Cancer Immunol Immunother 2015;64:1251-60.

15 Mahlendorf DE, Staege MS. Characterization of Ewing sarcoma associated cancer/testis antigens. Cancer Biol Ther 2013;14:254-61.

16 Morales $\mathrm{E}$, Olson M, Iglesias F, et al. Role of immunotherapy in Ewing sarcoma. J Immunother Cancer 2020;8:e000653.

17 Ohgami RS, Campagna DR, McDonald A, et al. The STEAP proteins are metalloreductases. Blood 2006;108:1388-94.

18 Korkmaz KS, Elbi C, Korkmaz CG, et al. Molecular cloning and characterization of STAMP1, a highly prostate-specific six transmembrane protein that is overexpressed in prostate cancer. $J$ Biol Chem 2002;277:36689-96.

19 Gomes IM, Maia CJ, Santos CR. Steap proteins: from structure to applications in cancer therapy. Molecular Cancer Research 2012;10:573-87.

20 Gomes IM, Maia CJ, Santos CR. Steap proteins: from structure to applications in cancer therapy. Mol Cancer Res 2012;10:573-87.

21 Wang L, Jin Y, Arnoldussen YJ, et al. Stamp1 is both a proliferative and an antiapoptotic factor in prostate cancer. Cancer Res 2010;70:5818-28.

22 Yang Q, Ji G, Li J. STEAP2 is down-regulated in breast cancer tissue and suppresses PI3K/Akt signaling and breast cancer cell invasion in vitro and in vivo. Cancer Biol Ther 2020;21:278-91.

23 Machlenkin A, Paz A, Bar Haim E, et al. Human CTL epitopes prostatic acid phosphatase- 3 and six-transmembrane epithelial antigen of prostate-3 as candidates for prostate cancer immunotherapy. Cancer Res 2005;65:6435-42.

24 Alves PMS, Faure O, Graff-Dubois S, et al. Steap, a prostate tumor antigen, is a target of human CD8+ T cells. Cancer Immunol Immunother 2006;55:1515-23.

25 Carrasquillo JA, Fine B, Pandit-Taskar N. Imaging metastatic castration-resistant prostate cancer patients with (89)Zr-DFOMSTP2109A anti-STEAP1 antibody. J Nucl Med 2019;60:1517-23.

26 Danila DC, Szmulewitz RZ, Vaishampayan U, et al. Phase I study of DSTP3086S, an antibody-drug conjugate targeting six-transmembrane epithelial antigen of prostate 1 , in metastatic castration-resistant prostate cancer. J Clin Oncol 2019;37:3518-27.

27 Barroca-Ferreira J, Pais JP, Santos MM, et al. Targeting STEAP1 protein in human cancer: current trends and future challenges. Curr Cancer Drug Targets 2018;18:222-30.

28 O'Donoghue JA, Danila DC, Pandit-Taskar N, et al. Pharmacokinetics and Biodistribution of a [ ${ }^{89} \mathrm{Zr}$ ]Zr-DFO-MSTP2109A Anti-STEAP1 
Antibody in Metastatic Castration-Resistant Prostate Cancer Patients. Mol Pharm 2019;16:3083-90.

29 Carrasquillo JA, Fine BM, Pandit-Taskar N, et al. Imaging Patients with Metastatic Castration-Resistant Prostate Cancer Using ${ }^{89}$ Zr-DFO-MSTP2109A Anti-STEAP1 Antibody. J Nucl Med 2019;60:1517-23.

$30 \mathrm{Xu} \mathrm{H}$, Cheng M, Guo H, et al. Retargeting T cells to GD2 pentasaccharide on human tumors using bispecific humanized antibody. Cancer Immunol Res 2015;3:266-77.

31 Cheng $\mathrm{M}$, Ahmed $\mathrm{M}$, Xu H, et al. Structural design of disialoganglioside GD2 and CD3-bispecific antibodies to redirect $T$ cells for tumor therapy. Int J Cancer 2015;136:476-86.

32 Lopez-Albaitero A, Xu H, Guo H, et al. Overcoming resistance to HER2-targeted therapy with a novel HER2/CD3 bispecific antibody. Oncoimmunology 2017;6:e1267891.

$33 \mathrm{Wu}$ Z, Guo H-F, Xu H, et al. Development of a tetravalent AntiGPA33/Anti-CD3 bispecific antibody for colorectal cancers. Mol Cancer Ther 2018:17:2164-75.

34 Nolan-Stevaux O. Abstract DDT02-03: AMG 509: a novel, humanized, half-life extended, bispecific STEAP1 1 CD3 T cell recruiting $X m A b \circledast 2+1$ antibody. Cancer Res 2020;80.

35 Santich BH, Park JA, Tran H, et al. Interdomain spacing and spatial configuration drive the potency of IgG-[L]-scFv T cell bispecific antibodies. Sci Trans/ Med 2020;12. doi:10.1126/scitransImed. aax1315. [Epub ahead of print: 1103 2020].

36 Wu Z, Cheung NV. T cell engaging bispecific antibody (T-BsAb): from technology to therapeutics. Pharmacol Ther 2018;182:161-75.

37 Challita-Eid PM, Morrison K, Etessami S, et al. Monoclonal antibodies to six-transmembrane epithelial antigen of the prostate-1 inhibit intercellular communication in vitro and growth of human tumor xenografts in vivo. Cancer Res 2007;67:5798-805.

38 Yu X, Pegram CN, Bigner DD, et al. Development and validation of a cell-based fluorescent method for measuring antibody affinity. $J$ Immunol Methods 2017:442:49-53.

$39 \mathrm{Xu} \mathrm{H}$, Cheng M, Guo H, et al. Retargeting T cells to GD2 pentasaccharide on human tumors using bispecific humanized antibody. Cancer Immunol Res 2015;3:266-77.

40 Jiao P, Otto M, Geng Q, et al. Enhancing both CT imaging and natural killer cell-mediated cancer cell killing by a GD2-targeting nanoconstruct. J Mater Chem B 2016;4:513-20.

41 Park JA, Cheung N-KV. Gd2 or HER2 targeting T cell engaging bispecific antibodies to treat osteosarcoma. $J$ Hematol Oncol 2020;13:172.

42 Zufferey R, Nagy D, Mandel RJ, et al. Multiply attenuated lentiviral vector achieves efficient gene delivery in vivo. Nat Biotechnol 1997; 15:871-5

43 Hoseini SS, Dobrenkov K, Pankov D, et al. Bispecific antibody does not induce T-cell death mediated by chimeric antigen receptor against disialoganglioside GD2. Oncoimmunology 2017;6:e1320625.

44 Wang L, Hoseini SS, Xu H, et al. Silencing Fc domains in T cellEngaging bispecific antibodies improves T-cell trafficking and antitumor potency. Cancer Immunol Res 2019;7:2013-24.

45 Ishiguro T, Sano Y, Komatsu S-I, et al. An anti-glypican 3/CD3 bispecific T cell-redirecting antibody for treatment of solid tumors. Sci Trans/ Med 2017;9. doi:10.1126/scitransImed.aal4291. [Epub ahead of print: 04 Oct 2017].

$46 \mathrm{Wu} \mathrm{Z}$, Guo H-F, Xu H, et al. Development of a tetravalent AntiGPA33/Anti-CD3 bispecific antibody for colorectal cancers. Mol Cancer Ther 2018;17:2164-75.
47 Gomes IM, Santos CR, Maia CJ. Expression of STEAP1 and STEAP1B in prostate cell lines, and the putative regulation of STEAP1 by post-transcriptional and post-translational mechanisms. Genes Cancer 2014:5:142-51.

48 Cheng M, Santich BH, Xu H, et al. Successful engineering of a highly potent single-chain variable-fragment (scFv) bispecific antibody to target disialoganglioside (GD2) positive tumors. Oncoimmunology 2016;5:e1168557.

49 Ahmed M, Cheng M, Cheung IY, et al. Human derived dimerization tag enhances tumor killing potency of a T-cell engaging bispecific antibody. Oncoimmunology 2015;4:e989776.

50 Owonikoko TK, Borghaei H, Champiat S, et al. Phase I study of AMG 757, a half-life extended bispecific T-cell engager (HLE bite immune therapy) targeting DLL3, in patients with small cell lung cancer (SCLC). Journal of Clinical Oncology 2020;38:TPS9080.

51 Park JA, Santich BH, Xu H, et al. Potent ex vivo armed T cells using recombinant bispecific antibodies for adoptive immunotherapy with reduced cytokine release. J Immunother Cancer 2021;9:e002222.

52 Cuesta Ángel M., Sainz-Pastor N, Bonet J, et al. Multivalent antibodies: when design surpasses evolution. Trends Biotechnol 2010;28:355-62.

53 Cheng M, Santich $\mathrm{BH}, \mathrm{Xu} \mathrm{H}$, et al. Successful engineering of a highly potent single-chain variable-fragment (scFv) bispecific antibody to target disialoganglioside (GD2) positive tumors. Oncoimmunology 2016;5:e1168557.

54 Wittrup KD, Thurber GM, Schmidt MM, et al. Practical theoretic guidance for the design of tumor-targeting agents. Methods Enzymol 2012;503:255-68.

55 Hoseini SS, Guo H, Wu Z, et al. A potent tetravalent T-cell-engaging bispecific antibody against CD33 in acute myeloid leukemia. Blood Adv 2018;2:1250-8.

56 Hoseini SS, Espinosa-Cotton M, Guo H-F, et al. Overcoming leukemia heterogeneity by combining $T$ cell engaging bispecific antibodies. J Immunother Cancer 2020;8:e001626.

57 Hoseini SS, Vadlamudi M, Espinosa-Cotton M, et al. T cell engaging bispecific antibodies targeting CD33 IgV and IgC domains for the treatment of acute myeloid leukemia. J Immunother Cancer 2021;9:e002509.

58 Cheung IY, Feng Y, Danis K, et al. Novel markers of subclinical disease for Ewing family tumors from gene expression profiling. Clin Cancer Res 2007;13:6978-83.

59 Grunewald TGP, Diebold I, Esposito I, et al. STEAP1 is associated with the invasive and oxidative stress phenotype of Ewing tumors. Mol Cancer Res 2012;10:52-65.

60 Majzner RG, Mackall CL. Tumor antigen escape from CAR T-cell therapy. Cancer Discov 2018;8:1219-26.

61 Casey DL, Lin T-Y, Cheung N-KV. Exploiting signaling pathways and immune targets beyond the standard of care for Ewing sarcoma. Front Oncol 2019;9:537.

62 Kedage V, Selvaraj N, Nicholas TR, et al. An interaction with Ewing's sarcoma breakpoint protein EWS defines a specific oncogenic mechanism of ETS factors rearranged in prostate cancer. Cell Rep 2016;17:1289-301.

63 Inlaseh-Catalano SM, Drigo SA, de Jesus CMN, et al. STEAP1 protein overexpression is an independent marker for biochemical recurrence in prostate carcinoma. Histopathology 2013;63:678-85.

64 Szewczyk M, Lechowski R, Zabielska K. What do we know about canine osteosarcoma treatment? review. Vet Res Commun 2015;39:61-7. 